Proceedings of the

International Geometry Center

Vol. 12, no. 4 (2019) pp. 1-49

\title{
Hamiltonian operators and related differential-algebraic Balinsky-Novikov, Riemann and Leibniz type structures on nonassociative noncommutative algebras
}

\author{
Orest D. Artemovych, Alexander A. Balinsky, \\ Anatolij K. Prykarpatski
}

\begin{abstract}
We review main differential-algebraic structures lying in background of analytical constructing multi-component Hamiltonian operators as derivatives on suitably constructed loop Lie algebras, generated by nonassociative noncommutative algebras. The related Balinsky-Novikov and Leibniz type algebraic structures are derived, a new nonassociative "Riemann" algebra is constructed, deeply related with infinite multi-component Riemann type integrable hierarchies. An approach, based on the classical Lie-Poisson structure on coadjoint orbits, closely related with those, analyzed in the present work and allowing effectively enough construction of Hamiltonian operators, is also briefly revisited. As the compatible Hamiltonian operators are constructed by means of suitable central extentions of the adjacent weak Lie algebras, generated by the right Leibniz and Riemann type nonassociative and noncommutative algebras, the problem of their description requires a detailed investigation both of their structural properties and finite-dimensional representations of the right Leibniz algebras defined by the corresponding structural constraints. Subject to these important aspects we stop in the work mostly on the structural properties of the right Leibniz algebras, especially on their derivation algebras and their generalizations. We have also added a short Supplement within which we revisited the classical Poisson manifold approach, closely related to our construction of Hamiltonian operators, generated by nonassociative and noncommutative algebras. In particular, we presented its natural and simple generalization allowing effectively to describe a wide class of Lax type integrable nonlinear Kontsevich type Hamiltonian systems on associative noncommutative algebras.
\end{abstract}

Keywords: Hamiltonian operators, Lie-Poisson structure, differenetial algebras, integrability, derivatives, loop-algebra, cocycles, Balinsky-Novikov algebra, right Leibniz algebra, Riemann algebra, group agebras, $\pi$-metrized Lie alegbras, Kontsevich type systems

DOI: http://dx.doi.org/10.15673/tmgc.v12i4.1554 


\begin{abstract}
Анотація. Дано огляд основних диференціально-алгебраїчних структур, що лежать в основі аналітичної побудови багато-компонентних операторів Гамільтона як диференціювань на відповідних алгебрах Лі петель, породжених неасоціативними некомутативними алгебрами. Введені алгебраїчні структури типу Балінського-Новікова і Ляйбница, побудована нова неасоціативна алгебра Рімана, глибоко пов'язана з нескінченними інтегровними багато-компонентними ієрархіями типу Рімана. Також коротко висвітлений підхід, що грунтується на класичних структурах ЛіПуассона на ко-приєднаних орбітах, які дозволяють ефективно конструювати оператори Гамільтона. Враховуючи, що узгоджені оператори Гамільтона генеруються відповідними центральними розширеннями приєднаних слабо-визначених алгебр Лі, породжених неасоціативними некомутативними алгебрами, проблема їх опису вимагає детального дослідження як структурних властивостей свойств, так і скінченно-вимірних зображень правих алгебр Ляйбніца, визначених відповідними структурними обмеженнями. Стосовно цих важливих аспектів ми обмежились в работі в основному правими алгебрами Лі, зокрема на їхніх алгебрах диференціювань та їх узагальненнях. Ми також помістили Додаток, в которому ми коротко висвітлили класичний підхід, що грунтується на пуассонових многовидах і тісно звязаний з нашою побудовою операторів Гамільтона, породженних неасоціативними некомутативними алгебрами. Зокрема, ми представили його природне та просте узагальнення, котре дозволяє ефективно будувати широкий клас нелінійних інтегровних за Лаксом гамільтонових систем типу Концевича на асоціативних некомутативних алгебрах.
\end{abstract}

\title{
1. INTRODUCTION
}

We present a short review of main differential-algebraic structures lying in background of analytical constructing multicomponent Hamiltonian operators as derivatives on suitably constructed loop Lie algebras, generated by nonassociative and noncommutative algebras. During the last decades there were discovered [38, 22, 20, 70] many integrable Hamiltonian systems, whose internal symmetyry structure was analytical nature was understood owing to the Lie-algebraic properties of their internal hidden symmetry structures. A first account of the Hamiltonian operators and related differential-algebraic structures, lying in the background of integrable systems, was given by I. Gelfand and I. Dorfman [43, 34] and later was extended by B. Dubrovin and S. Novikov [36, 37], and also by A. Balinsky and S. Novikov [11, 14, 12, 13]. Also some new special differential-algebraic techniques [80] were devised for studying the Lax integrability and the structure of related Hamiltonian operators for a wide class of the Riemann type hydrodynamic hierarchies. Just recently considerable work $[8,10,9,73]$ has been done devoted to the finite dimensional representations of the Balinsky-Novikov algebra. Their importance 
for constructing integrable multi-component nonlinear Camassa-Holm type dynamical systems on functional manifolds was demonstrated by I. Strachan and B. Szablikowski in [91], which in part suggested the Lie-algebraic imbedding of the Balinsky-Novikov algebra into the general Lie-Poisson orbits scheme of classifying Lax integrable Hamiltonian systems. It is also worth of mentioning the related work [46] by Holm and Ivanov in which integrable multicomponent nonlinear Camassa-Holm type dynamical systems on functional manifolds were constructed.

In our work here we describe a differential-algebraic reformulation of the classical Lie algebraic scheme and develop an effective approach to classification of the underlying algebraic structures of integrable multicomponent Hamiltonian systems. In particular, we have devised a simple algorithm allowing to construct new algebraic structures within which the corresponding Hamiltonian operators exist and generate integrable multicomponent dynamical systems. We show, as examples, that the well-known BalinskyNovikov algebraic structure, obtained in $[43,11]$ as a condition for a matrix differential expression to be Hamiltonian and in [19, 27, 50,61] as a flat torsion free left-invariant affine connection on affine manifolds, affine structures and convex homogeneous cones, appears in our approach as a derivation on the Lie-algebra naturally associated with a suitably constructed differential loop algebra. As a direct generalization of this example we obtain two new differentiations, whose underlying algebraic structures coincide, respectively, with the well-known $[3,40]$ right Leibniz algebra, introduced in $[23,24,59]$, and with a new "Riemann" algebra, which naturally generate different Hamiltonian operators describing a wide class of multicomponent hierarchies $[21,80]$ of integrable Riemann hydrodynamic systems. As the compatible Hamiltonian operators, important for studying integrable multicomponent Hamiltonian systems, are constructed by means of suitable central extentions of the adjacent weak Lie algebras, determined by the right Leibniz and Riemann type nonassociative and noncommutative algebras, their description requires a detailed investigation both of the structural properties and finite-dimensional representations of the right Leibniz algebras defined by the corresponding structural constraints. Subject to these important aspects we stop in the work mostly on the structural properties of the right Leibniz algebras, especially on their derivation algebras and their generalizations.

In a supplement the classical Poisson manifold approach, closely related to our construction of Hamiltonian operators, generated by nonassociative and noncommutative algebras, is briefly revisited. In particular, its natural and simple generalization appeared to be useful $[5,6,94,96,62,66,67]$ for 
describing a wide class of Lax type integrable nonlinear Hamiltonian systems on associative noncommutative algebras, initiated first in $[25,35,76$, 78 in case of the noncommutative operator algebras and continued later in $[62,51,52,53,62,66,67,71]$ in case of general associative noncommutative algebras.

\section{The Hamiltonian operators and Related Algebraic STRUCTURES VIA THE DIFFERENTIAL-ALGEBRAIC APPROACH}

Let $(\mathbb{A} ; \circ)$ be a finite-dimensional algebra of dimension $N=\operatorname{dim} \mathbb{A} \in \mathbb{Z}_{+}$ (in general noncommutive and nonassociative) over an algebraically closed field $\mathbb{K}$. Using the algebra $\mathbb{A}$ one can construct the related loop algebra $\widetilde{\mathbb{A}}$ of smooth mappings $u: \mathbb{S}^{1} \rightarrow \mathbb{A}$ and endow it with a suitably generalized natural convolution $\langle\cdot, \cdot\rangle$ on $\widetilde{\mathbb{A}}^{*} \times \widetilde{\mathbb{A}} \rightarrow \mathbb{K}$, where $\widetilde{\mathbb{A}}^{*}$ is the corresponding adjoint to $\widetilde{\mathbb{A}}$ space.

First, we shall consider a general scheme of constructing nontrivial ultralocal and local [38] Poisson structures on the adjoint space $\widetilde{\mathbb{A}}^{*}$, compatible with the internal multiplication in the loop algebra $\widetilde{\mathbb{A}}$. Consider a basis $\left\{e_{s} \in \mathbb{A}: s=\overline{1, N}\right\}$ of the algebra $\mathbb{A}$ and its dual $\left\{u^{s} \in \mathbb{A}^{*}: s=\overline{1, N}\right\}$ with respect to $\langle\cdot, \cdot\rangle$ on $\mathbb{A}^{*} \times \mathbb{A}$, that is $\left\langle u^{j}, e_{i}\right\rangle:=\delta_{i}^{j}$ for all $i, j=\overline{1, N}$, and such that for any $u(x)=\sum_{s=\overline{1, N}} u_{s}(x) u^{s} \in \widetilde{\mathbb{A}}^{*}, x \in \mathbb{S}^{1}$, the quantities $u_{s}(x):=\left\langle u(x), e_{s}\right\rangle \in \mathbb{K}$ for all $s=\overline{1, N}, x \in \mathbb{S}^{1}$. Put

$$
\widetilde{\mathbb{A}} \wedge \widetilde{\mathbb{A}}:=\operatorname{Skew}(\widetilde{\mathbb{A}} \otimes \widetilde{\mathbb{A}})
$$

and let $\vartheta^{*}: \widetilde{\mathbb{A}} \wedge \widetilde{\mathbb{A}} \rightarrow \widetilde{\mathbb{A}}$ be a skew-symmetric bilinear mapping. Then the expression

$$
\left\{u_{i}(x), u_{j}(x)\right\}:=\left\langle u(x), \vartheta^{*}\left(e_{i} \wedge e_{j}\right)\right\rangle
$$

defines for any $x, y \in \mathbb{S}^{1}$ and all $i, j=\overline{1, N}$ an ultra-local linear skewsymmetric pre-Poisson bracket on $\widetilde{\mathbb{A}}^{*}$. Since the algebra $\widetilde{\mathbb{A}}$ possesses its internal multiplicative structure "o", the important problem arises: Under what conditions is the pre-Poisson bracket (2.1) Poisson and compatible with this internal structure on $\widetilde{\mathbb{A}}$ ? To proceed with elucidating this question, we define a co-multiplication $\Delta: \widetilde{\mathbb{A}}^{*} \rightarrow \widetilde{\mathbb{A}}^{*} \otimes \widetilde{\mathbb{A}}^{*}$ on any element $u \in \widetilde{\mathbb{A}}^{*}$ by means of the relationship

$$
\langle\Delta(u),(a \otimes b)\rangle:=\langle u, a \circ b\rangle
$$

for arbitrary $a, b \in \widetilde{\mathbb{A}}$. Note that the co-multiplication $\Delta: \widetilde{\mathbb{A}}^{*} \rightarrow \widetilde{\mathbb{A}}^{*} \otimes \widetilde{\mathbb{A}}^{*}$, defined this way, is a homomorphism of the algebra $\widetilde{\mathbb{A}}^{*}$ with respect to the natural multiplication of functionals, and the linear pre-Poisson structure 
$\{\cdot, \cdot\}(2.1)$ on $\widetilde{\mathbb{A}}^{*}$ is called compatible with the multiplication "o" on the algebra $\widetilde{\mathbb{A}}$, if the following symbolic invariance condition

$$
\Delta\left\{u_{i}(x), u_{j}(x)\right\}=\left\{\Delta\left(u_{i}(x)\right), \Delta\left(u_{j}(x)\right)\right\}
$$

holds for any $x \in \mathbb{S}^{1}$ and all $i, j=\overline{1, N}$.

Taking into account that multiplication in the algebra $\mathbb{A}$ is given for any $i, j=\overline{1, N}$ by the condition

$$
e_{i} \circ e_{j}:=\sum_{s=\overline{1, N}} \sigma_{i j}^{s} e_{s},
$$

where the quantities $\sigma_{i j}^{s} \in \mathbb{K}$ for all $i, j$ and $k=\overline{1, N}$ are constants, the related co-multiplication $\Delta: \widetilde{\mathbb{A}}^{*} \rightarrow \widetilde{\mathbb{A}}^{*} \otimes \widetilde{\mathbb{A}}^{*}$ acts on the basic functionals $u^{s} \in \widetilde{\mathbb{A}}^{*}, s=\overline{1, N}$, as

$$
\Delta\left(u^{s}\right)=\sum_{i, j=\overline{1, N}} \sigma_{i j}^{s} u^{i} \otimes u^{j}
$$

Additionally, if the mapping $\vartheta^{*}: \widetilde{\mathbb{A}} \wedge \widetilde{\mathbb{A}} \rightarrow \widetilde{\mathbb{A}}$ is given, for instance, in the simple linear form

$$
\vartheta^{*}:\left(e_{i} \otimes e_{j}-e_{j} \otimes e_{i}\right) \rightarrow \sum_{s=\overline{1, N}}\left(c_{i j}^{s}-c_{j i}^{s}\right) e_{s},
$$

where quantities $c_{i j}^{s} \in \mathbb{K}$ are constant for all $i, j$ and $s=\overline{1, N}$, then for the adjoint to $(2.6)$ mapping $\vartheta: \operatorname{Symm}(\widetilde{\mathbb{A}} *) \rightarrow \widetilde{\mathbb{A}}^{*} \wedge \widetilde{\mathbb{A}}^{*}$ one obtains the expression

$$
\vartheta: u^{s} \rightarrow \sum_{i, j=\overline{1, N}}\left(c_{i j}^{s}-c_{j i}^{s}\right) u^{i} \otimes u^{j} .
$$

For the pre-Poisson bracket $(2.1)$ to be a Poisson bracket on $\widetilde{\mathbb{A}}^{*}$, it should must also satisfy the Jacobi identity. To find the corresponding additional constraints on the internal multiplication "O" on the algebra $\widetilde{\mathbb{A}}$, define for any $u(x) \in \widetilde{\mathbb{A}}^{*}$ the skew-symmetric linear mapping

$$
\vartheta(u): \widetilde{\mathbb{A}} \rightarrow \widetilde{\mathbb{A}}^{*},
$$

called [43] by the Hamiltonian operator, via the identity

$$
\langle\vartheta(u) a, b\rangle:=\langle\vartheta u(x), a \wedge b\rangle
$$

for any $a, b \in \widetilde{\mathbb{A}}$, where the mapping $\vartheta: \widetilde{\mathbb{A}}^{*} \rightarrow \widetilde{\mathbb{A}}^{*} \wedge \widetilde{\mathbb{A}}^{*}$ is determined by the expression (2.7) to which it is adjoint. Then it is well known [43] that the pre-Poisson bracket (2.1) is Poisson iff the Hamiltonian operator (2.8) satisfies the well known [43] Schouten- $\sigma$ Nijenhuis condition:

$$
[[\vartheta(u), \vartheta(u)]]=0
$$


for any $u(x) \in \widetilde{\mathbb{A}}^{*}$. Since the mapping

$$
\vartheta(u) e_{i}=\sum_{s, k=\overline{1, N}}\left(c_{i k}^{s}-c_{k i}^{s}\right) u_{s}(x) u^{k}
$$

holds for any basis element $e_{i} \in \mathbb{A}, i=\overline{1, N}$, the resulting linear pre-Poisson bracket (2.1) becomes equal to

$$
\begin{aligned}
\left\{u_{i}(x), u_{j}(x)\right\} & =\left\langle\vartheta(u) e_{i}, e_{j}\right\rangle=\sum_{s=\overline{1, N}}\left(c_{i j}^{s}-c_{j i}^{s}\right) u_{s}(x) \\
& =\left\langle u(x), \sum_{s=\overline{1, N}}\left(c_{i j}^{s}-c_{j i}^{s}\right) e_{s}\right\rangle
\end{aligned}
$$

for any $u(x) \in \widetilde{\mathbb{A}}^{*}$. Now, defining on the algebra $\mathbb{A}$ the naturally adjacent to the algebra $\mathbb{A}$ Lie commutator structure

$$
\left[e_{i}, e_{j}\right]=e_{i} \circ e_{j}-e_{j} \circ e_{i}:=\sum_{s=\overline{1, N}}\left(\sigma_{i j}^{s}-\sigma_{j i}^{s}\right) e_{s}
$$

for any basis elements $e_{i}, e_{j} \in \mathbb{A}, i, j=\overline{1, N}$, the expression (2.12) yields the well known [1, 4] classical Lie-Poisson bracket

$$
\left\{u_{i}(x), u_{j}(x)\right\}=\left\langle u,\left[e_{i}, e_{j}\right]\right\rangle .
$$

Concerning the adjacent Lie algebra structure condition (2.13), it can be easily rewritten as the set of relationships,

$$
\sigma_{i j}^{s}-\sigma_{j i}^{s}=c_{i j}^{s}-c_{j i}^{s}
$$

whose evident solution is

$$
c_{i j}^{s}=\sigma_{i j}^{s}
$$

for any $i, j, s=\overline{1, N}$. It is also clear that the compatibility condition (2.3) is completely unnecessary $[14,12]$ for the pre-Poisson bracket $(2.1)$ to be a Poisson one. Moreover, as the bracket (2.14) is of the classical Lie-Poisson type, for the Hamiltonian operator (2.11) to satisfy the Schouten-Nijenhuis condition (2.10) is enough to check only the weak Jacobi identity for the weak Lie algebra $\mathcal{L}_{\widetilde{\mathbb{A}}}$, adjacent to to the algebra $\widetilde{\mathbb{A}}$ via imposing the Lie structure (2.13), taking into account the relationships (2.16). Simple calculations for the special skew-symmetric case

$$
e_{i} \circ e_{j}+e_{j} \circ e_{i}=0
$$

for all $i, j=\overline{1, N}$ give rise to the constraints

$$
e_{i} \circ e_{j}+e_{j} \circ e_{i}=0,\left(e_{i} \circ e_{j}\right) \circ e_{k}+\left(e_{j} \circ e_{k}\right) \circ e_{i}+\left(e_{k} \circ e_{i}\right) \circ e_{j}=0,
$$


coinciding exactly with those in [43]. The corresponding Hamiltonian operator (2.8) then acts as

$$
\vartheta(u) e_{i}=\sum_{s, k=\overline{1, N}}\left(\sigma_{i k}^{s}-\sigma_{k i}^{s}\right) u_{s}(x) u^{k}
$$

on any basis element $e_{i} \in \mathbb{A}$. Since the bracket (2.14), owing to the constraints (2.17) and (2.18), satisfies the weak Jacobi identity implying that the mapping $\vartheta(u): \widetilde{\mathbb{A}} \rightarrow \widetilde{\mathbb{A}}^{*}$ satisfies the Schouten-Nijenhuis condition (2.10), one has the following result.

Theorem 2.1. The general linear pre-Poisson bracket (2.1) on $\widetilde{\mathbb{A}}^{*}$ under the constraints (2.17) and (2.18) on the algebra $\mathbb{A}$, which is of the LiePoisson type on the adjoint space $\mathcal{L}_{\widetilde{\mathbb{A}}}^{*}$ to the weak Lie algebra $\mathcal{L}_{\widetilde{\mathbb{A}}}$ adjacent to the loop algebra $\widetilde{\mathbb{A}}$, is a priori a Poisson and compatible with the internal algebraic structure of $\mathbb{A}$.

Remark 2.2. Similarly, one can consider a simple ultra-local quadratic pre-Poisson bracket on $\widetilde{\mathbb{A}}^{*}$,

$$
\left\{u_{i}(x), u_{j}(x)\right\}:=\left\langle u(x) \otimes u(x), \vartheta^{*}\left(e_{i} \wedge e_{j}\right)\right\rangle,
$$

where the skew-symmetric mapping $\vartheta^{*}: \widetilde{\mathbb{A}} \wedge \widetilde{\mathbb{A}} \rightarrow \operatorname{Symm}(\widetilde{\mathbb{A}} \otimes \widetilde{\mathbb{A}})$ is given for any $i, j=\overline{1, N}$ in the quadratic form

$$
\vartheta^{*}\left(e_{i} \otimes e_{j}-e_{j} \otimes e_{i}\right):=\sum_{k, s=\overline{1, N}}\left(c_{i j}^{k s}-c_{j i}^{k s}\right)\left(e_{k} \otimes e_{s}+e_{s} \otimes e_{k}\right) \text {. }
$$

In particular, if we assume that the coefficients $c_{i j}^{k s}=\sigma_{i j}^{k} \alpha^{s}$ for some constant numbers $\sigma_{i j}^{k}$ and $\alpha^{s} \in \mathbb{K}$ for all $i, j$ and $k, s=\overline{1, N}$, where

$$
e_{k} \circ e_{s}:=\sum_{k=\overline{1, N}} \sigma_{i j}^{k} e_{k},
$$

then the pre-Poissson bracket (2.20) yields a very compact form

$$
\left\{u_{i}(x), u_{j}(x)\right\}:=\left\langle u(x) \otimes u(x), \alpha \otimes\left[e_{i}, e_{j}\right]+\left[e_{i}, e_{j}\right] \otimes \alpha\right\rangle,
$$

generalizing (2.14) and parametrically depending on the constant vector $\alpha:=\sum_{s=\overline{1, N}} \alpha^{s} e_{s} \in \mathbb{A}$. For the pre-Poisson bracket (2.22) to be Poisson one can formulate suitable constraints on the algebraic structure of $\widetilde{\mathbb{A}}$, similar to those obtained in [14], which we shall not consider here.

Now, let $\widetilde{\mathbb{A}}(u) \subset \widetilde{\mathbb{A}}$ denote the polynomial differential ideal generated by an element $u \in \widetilde{\mathbb{A}}$ and its derivatives $D_{x}^{n} u \in \widetilde{\mathbb{A}}, n \in \mathbb{Z}_{+}$. The corresponding space of polynomial functions $\widetilde{\mathbb{A}}(u) \rightarrow \mathbb{K}$, constructed by means of some 
scalar form on $\widetilde{\mathbb{A}}(u)$, will be denoted by $\mathcal{F}_{\widetilde{\mathbb{A}}}(u)$. Then the basic ultra-local and linear, with respect to an independent element $u(x) \in \widetilde{\mathbb{A}}, x \in \mathbb{S}^{1}$, prePoisson bracket (2.1) is easily generalized to a local pre-Poisson bracket for arbitrary functions $f, g \in \mathcal{F}_{\widetilde{\mathbb{A}}}(u)$ :

$$
\{f, g\}(u)=\left\langle u(x), \vartheta^{*}(\nabla f(u(x)) \wedge \nabla g(u(x)\rangle,\right.
$$

in which the mapping

$$
\vartheta^{*}: \widetilde{\mathbb{A}} \wedge \widetilde{\mathbb{A}} \rightarrow \operatorname{Symm}(\widetilde{\mathbb{A}} \otimes \widetilde{\mathbb{A}})
$$

is invariantly reduced on the subspace $\widetilde{\mathbb{A}}(u) \wedge \widetilde{\mathbb{A}}(u)$ and depends nontrivially on the derivation $D_{x}: \widetilde{\mathbb{A}} \rightarrow \widetilde{\mathbb{A}}$. In $(2.23)$ we denoted the usual linear gradient mapping from $\mathcal{F}_{\widetilde{\mathbb{A}}}(u)$ to the ideal $\widetilde{\mathbb{A}}(u) \subset \widetilde{\mathbb{A}}$ by " $\nabla$ ", that is for a given $h \in \mathcal{F}_{\widetilde{\mathbb{A}}}(u), \nabla h(u(x)) \in \widetilde{\mathbb{A}}(u)$ and

$$
\left\langle v(x), \nabla h(u(x)\rangle:=d h(u+\varepsilon v) /\left.d \varepsilon\right|_{\varepsilon=0}\right.
$$

for any $v(x) \in \widetilde{\mathbb{A}}^{*}, x \in \mathbb{S}^{1}$. Keeping in mind the problem of finding constraints on the multiplicative structure of the algebra $\widetilde{\mathbb{A}}$ under which the pre-Poisson bracket (2.23) is Poisson, it is very interesting to construct nontrivial examples of linear local pre-Poisson brackets on $\mathcal{F}_{\widetilde{\mathbb{A}}}(u)$, compatible with the multiplication "o" on $\mathbb{A}$ and nontrivially depending on the usual differential operator $D_{x}: \widetilde{\mathbb{A}} \rightarrow \widetilde{\mathbb{A}}$ for $x \in \mathbb{S}^{1}$.

In particular, for arbitrary functions $f, g \in \mathcal{F}_{\widetilde{\mathbb{A}}}(u)$ one can consider the following nontrivial and simplest linear local pre-Poisson bracket

$$
\{f, g\}(u):=\left\langle u(x), \vartheta^{*}(\nabla f(u(x)) \wedge \nabla g(u(x))\rangle,\right.
$$

where

$$
\begin{aligned}
\vartheta^{*}:(a(x) \wedge b(x)) & \longrightarrow \\
\longrightarrow & \sum_{j, k, s=\overline{1, N}}\left[c_{j k}^{s} D_{x} a^{j}(x) b^{k}(x)-c_{j k}^{s} D_{x} b^{j}(x) a^{k}(x)\right] e_{s}
\end{aligned}
$$

for any

$$
a(x):=\sum_{j=\overline{1, N}} a^{j}(x) e_{j}, b(x):=\sum_{j=\overline{1, N}} b^{j}(x) e_{j} \in \widetilde{\mathbb{A}},
$$

$x \in \mathbb{S}^{1}$, and some arbitrarily chosen constant quantities $c_{j k}^{s} \in \mathbb{K}$ for all $j, k$ and $s=\overline{1, N}$. If one also assumes that these constant quantities satisfy the condition (2.16), that is $c_{i j}^{s}=\sigma_{i j}^{s}$ for all $i, j$ and $s=\overline{1, N}$, the mapping (2.25) can be recast as

$$
\vartheta^{*}:(a(x) \wedge b(x)) \longrightarrow D_{x} a(x) \circ b(x)-D_{x} b(x) \circ a(x),
$$


providing the pre-Poisson bracket (2.24) for arbitrary functions $f, g \in \mathcal{F}_{\widetilde{\mathbb{A}}}(u)$ with the canonical Lie-Poisson form

$$
\begin{aligned}
\{f, g\}(u):=\left\langle u(x), D_{x} \nabla f(u(x)) \circ \nabla g(\right. & u(x))- \\
& -D_{x} \nabla g(u(x)) \circ \nabla f(u(x)\rangle,
\end{aligned}
$$

which was recently presented in [91]. Thus, if the Lie structure

$$
[a(x), b(x)]_{D}:=D_{x} a(x) \circ b(x)-D_{x} b(x) \circ a(x)
$$

for any $a(x), b(x) \in \widetilde{\mathbb{A}}, x \in \mathbb{S}^{1}$, generates the weak adjacent Lie algebra $\mathcal{L}_{\widetilde{\mathbb{A}}}$, the pre-Poisson bracket (2.27) will automatically be Poisson on the space $\mathcal{F}_{\widetilde{\mathbb{A}}}(u)$. Moreover, the expression $(2.27)$, rewritten in the tensor form

$$
\begin{aligned}
\{f, g\}(u) & =\left\langle\Delta u(x), D_{x} \nabla f(u(x)) \otimes \nabla g(u(x))-\right. \\
& -D_{x} \nabla g(u(x)) \otimes \nabla f(u(x)\rangle= \\
& :=(\vartheta(u) \nabla f(u(x)), \nabla g(u(x))),
\end{aligned}
$$

naturally defines a related bilinear form $(\cdot, \cdot)$ on the weak adjacent Lie algebra $\mathcal{L}_{\widetilde{\mathbb{A}}}$, allowing to determine the corresponding Hamiltonian operator $\vartheta(u): \mathcal{L}_{\widetilde{\mathbb{A}}} \rightarrow \mathcal{L}_{\widetilde{\mathbb{A}}}$, whose matrix representation with respect to the basis $\left\{e_{s} \in \mathbb{A} \mid s=\overline{1, N}\right\}$ is

$$
\vartheta(u)=\sigma(u) D_{x}+D_{x} \sigma(u)^{\top},
$$

where $\sigma(u):=\left\{\sum_{s=\overline{1, N}} \sigma_{i j}^{s} u_{s} \mid i, j=\overline{1, N}\right\}$. So, if the Hamiltonian operator (2.30) satisfies the Schouten-Nijenhuis condition (2.10), the pre-Poisson bracket (2.29) is Poisson. Yet, simultaneously, if the adjacent Lie algebra structure (2.28) satisfies the weak Jacobi condition

$$
\begin{aligned}
& \left\langle u(x),\left[[a(x), b(x)]_{D}, c(x)\right]_{D}\right\rangle \\
& +\left\langle u(x),\left[[b(x), c(x)]_{D}, a(x)\right]_{D}\right\rangle+ \\
& \quad+\left\langle u(x),\left[[c(x), a(x)]_{D}, b(x)\right]_{D}\right\rangle=0
\end{aligned}
$$

for any elements $a(x), b(x)$ and $c(x) \in \widetilde{\mathbb{A}}, x \in \mathbb{S}^{1}$, then the pre-Poisson bracket (2.27) equivalent to (2.29), being of the Lie-Poisson type, will be $a$ priori Poisson. As the second criterion is easier to check, after some simple calculations one obtains the well-known [11, 43] Balinsky-Novikov algebra constraints

$$
\left[R_{a}, R_{b}\right]=0,\left[L_{a}, L_{b}\right]=L_{[a, b]}
$$

on the multiplication structure of the algebra $\mathbb{A}$, where, by definition, for any $a, b \in \mathbb{A}$ the bracket $[a, b]:=a \circ b-b \circ a$ and the mappings $L_{a}, R_{a}: \mathbb{A} \rightarrow \mathbb{A}$ are left and right multiplications, respectively: $L_{a} b:=a \circ b=R_{b} a$, 
Remark 2.3. As follows from Proposition 3.2, formulated below, if the algebra $\mathbb{A}$ is a Balinsky-Novikov algebra (2.32), then the constructed above Hamiltonian operator $\vartheta(u): \mathcal{L}_{\widetilde{\mathbb{A}}} \rightarrow \mathcal{L}_{\widetilde{\mathbb{A}}}$ is a derivation of the weak adjacent Lie algebra $\mathcal{L}_{\widetilde{\mathbb{A}}}$ and vice versa: the operator $\vartheta(u): \mathcal{L}_{\widetilde{\mathbb{A}}} \rightarrow \mathcal{L}_{\widetilde{\mathbb{A}}}$ is Hamiltonian if it is a derivation of the weak Lie algebra $\mathcal{L}_{\widetilde{\mathbb{A}}}$ adjacent to the BalinskyNovikov algebra (2.32).

The next example of the bilinear, nonlocal (pseudodifferential) and weakly skew-symmetric mapping

$$
\vartheta^{*}:(a(x) \wedge b(x)) \rightarrow D_{x}^{-1} a(x) \circ b(x)-D_{x}^{-1} b(x) \circ a(x),
$$

where $D_{x} D_{x}^{-1}:=1: \widetilde{\mathbb{A}} \rightarrow \widetilde{\mathbb{A}}$ is the identity mapping, generates the weak adjacent Lie algebra $\mathcal{L}_{\widetilde{\mathbb{A}}}$ structure

$$
[a(x), b(x)]_{D}:=D_{x}^{-1} a(x) \circ b(x)-D_{x}^{-1} b(x) \circ a(x)
$$

for any $a(x), b(x) \in \widetilde{\mathbb{A}}$. It is easy to check that the commutator structure (2.34) satisfies the weak Jacobi identity (2.31) iff the multiplicative structure of the algebra $\mathbb{A}$ satisfies the so called [59] right Leibniz algebra constraints:

$$
R_{a \circ b}=\left[R_{a}, R_{b}\right], R_{a \circ b}+R_{b \circ a}=0
$$

for arbitrary elements $a, b \in \mathbb{A}$. The corresponding matrix integro-differential Hamiltonian operator on the space $\mathcal{F}_{\widetilde{\mathbb{A}}}(u)$ with respect to the basis

$$
\left\{e_{s} \in \mathbb{A} \mid s=\overline{1, N}\right\}
$$

for this case equals

$$
\vartheta(u)=\sigma(u) D_{x}^{-1}+D_{x}^{-1} \sigma(u)^{\top}
$$

for any $u(x) \in \widetilde{\mathbb{A}}^{*}, x \in \mathbb{S}^{1}$.

Consider now the bilinear, nonlocal and weakly skew-symmetric mapping

$$
\left.\left.\vartheta^{*}:(a(x) \wedge b(x)) \rightarrow D_{x}^{-1} b(x) \circ D_{x} a(x)\right)-D_{x}^{-1} a(x) \circ D_{x} b(x)\right),
$$

which naturally generates the adjacent Lie algebra $\mathcal{L}_{\widetilde{\mathbb{A}}}$ structure

$$
[a(x), b(x)]_{D}:=D_{x}^{-1} b(x) \circ D_{x} a(x)-D_{x}^{-1} a(x) \circ D_{x} b(x) .
$$

Then it is easy to check that the commutator structure (2.38) satisfies the weak Jacobi identity (2.31), iff the following so called Riemann algebra $\mathbb{A}$ multiplicative structure

$$
\left[R_{a}, R_{b}\right]=0, \quad L_{a \circ b}=R_{a \circ b}=L_{b \circ a}
$$

holds for arbitrary elements $a, b \in \mathbb{A}$. For the related Hamiltonian operator on the functional space $\mathcal{F}_{\widetilde{\mathbb{A}}}(u)$ with respect to the basis $\left\{e_{s} \in \mathbb{A} \mid s=\overline{1, N}\right\}$ one easily obtains from (2.37) the integro-differential expression

$$
\vartheta(u)=D_{x} \sigma(u) D_{x}^{-1}-D_{x}^{-1} \sigma(u)^{\top} D_{x}
$$


for any $u(x) \in \widetilde{\mathbb{A}}^{*}, x \in \mathbb{S}^{1}$. The above results can be reformulated as the following theorem.

Theorem 2.4. An arbitrary linear pre-Poisson bracket (2.29) on the functional space $\mathcal{F}_{\widetilde{\mathbb{A}}}(u)$, which is of the Lie-Poisson type on the adjoint space $\mathcal{L}_{\widetilde{\mathbb{A}}}^{*}$ to the weak Lie algebra $\mathcal{L}_{\widetilde{\mathbb{A}}}$ adjacent to the loop algebra $\widetilde{\mathbb{A}}$, is a priori Poisson and compatible with the internal structure of the algebra $\mathbb{A}$ iff the related Lie algebra structure $[\cdot, \cdot]_{D}$ satisfies the weak Jacobi condition.

Thus, all the operators $(2.30),(2.36)$ and (2.40) are Hamiltonian and $a$ priori satisfy the Schouten-Nijenhuis condition (2.10), as easily follows from Theorem 2.4. It is also clear that in contrast to the simple Hamiltonian operator criterion formulated in this theorem, direct and very cumbersome checking of the Schouten-Nijenhuis condition as in [43] for the cases considered above, would be required for the multiplicative structures on the algebra $\mathbb{A}$ coinciding with (2.32), (2.35) and (2.39).

\section{Hamiltonian operators AND RELATEd ALGEBraic STRUCTURES VIA THE LIE-ALGEBRAIC APPROACH}

Assume now that the loop algebra $(\widetilde{\mathbb{A}} ; \circ)$ allows a weak adjacent Lie algebra extension $\left(\mathcal{L}_{\widetilde{\mathbb{A}}} ;[\cdot, \cdot]_{D}\right)$ by means of a commutator operation

$$
[\cdot, \cdot]_{D}: \widetilde{\mathbb{A}} \times \widetilde{\mathbb{A}} \rightarrow \widetilde{\mathbb{A}}
$$

which can be endowed $[17,18,38,89]$ with a nondegenerate ad-invariant

$$
\left([a, b]_{D}, c\right)=\left(a,[b, c]_{D}\right)
$$

and symmetric

$$
(a, b)=(b, a)
$$

bilinear form $(\cdot, \cdot): \mathcal{L}_{\widetilde{\mathbb{A}}} \times \mathcal{L}_{\widetilde{\mathbb{A}}} \rightarrow \mathbb{K}$ for any $a, b$ and $c \in \mathcal{L}_{\mathbb{A}} \cdot$

Remark 3.1. If the Lie-structure $[\cdot, \cdot]_{D}$ on $\widetilde{\mathbb{A}}$ coincides with the usual commutator structure $[\cdot, \cdot]$ on $\widetilde{\mathbb{A}}$, with respect to the multiplication "॰" and the symmetric bilinear form (3.2) also satisfies the shifting property

$$
(a \circ b, c)=(a, b \circ c)
$$

for any $a, b$ and $c \in \mathcal{L}_{\widetilde{\mathbb{A}}}$, then the ad-invariance condition (3.1) automatically holds.

The form (3.2) makes it possible to construct the natural identification $\mathcal{L}_{\widetilde{\mathbb{A}}}^{*} \simeq \mathcal{L}_{\widetilde{\mathbb{A}}}$. One can consider the subspace of polynomial functions $\mathcal{F}_{\widetilde{\mathbb{A}}}(u)$ 
of the space $\mathcal{F}\left(\mathcal{L}_{\widetilde{\mathbb{A}}}^{*}\right)$ of smooth functions on $\mathcal{L}_{\widetilde{\mathbb{A}}}^{*}$, generated by an element $u \in \mathcal{L}_{\widetilde{\mathbb{A}}}^{*}$, jointly with its related Lie-Poisson bracket:

$$
\{f, g\}_{0}:=\left(u,[\nabla f(u), \nabla g(u)]_{D}\right)
$$

for any $f, g \in \mathcal{F}_{\widetilde{\mathbb{A}}}(u)$. Owing to the construction $[1,4,20,17,38]$, the LiePoisson bracket (3.4) satisfies a priori the classical Jacobi identity, and it can serve as a very powerful tool for constructing the related Hamiltonian operators on the functional space $\mathcal{F}_{\widetilde{\mathbb{A}}}(u)$. In particular, following $[43,68]$, a smooth mapping $\vartheta(u): \mathcal{L}_{\widetilde{\mathbb{A}}} \rightarrow \mathcal{L}_{\widetilde{\mathbb{A}}}^{*} \simeq \mathcal{L}_{\widetilde{\mathbb{A}}}$ for a chosen element $u \in \widetilde{\mathbb{A}}$ is a Hamiltonian operator if the related pre-Poisson bracket

$$
\{f, g\}:=(\vartheta(u) \nabla f(u), \nabla g(u)),
$$

determined for any $f, g \in \mathcal{F}_{\widetilde{\mathbb{A}}}(u)$, satisfies the Jacobi identity.

Taking into account that the canonical Lie-Poisson bracket (3.4) depends essentially on the loop Lie algebra structure of $\mathcal{L}_{\widetilde{\mathbb{A}}}$, we proceed further to extending the Lie algebra structure on $\mathcal{L}_{\widetilde{\mathbb{A}}}$ by means of the standard [38] central extension technique. Namely, let $\hat{\mathcal{L}}_{\widetilde{\mathbb{A}}}:=\mathcal{L}_{\widetilde{\mathbb{A}}} \oplus \mathbb{K}$ denote the centrally extended Lie algebra $\mathcal{L}_{\widetilde{\mathbb{A}}}$ endowed with the bracket

$$
[(a ; \alpha),(b ; \beta)]_{D}:=\left([a, b]_{D} ; \varpi_{2}(a, b)\right)
$$

for any $a, b \in \mathcal{L}_{\widetilde{\mathbb{A}}}$ and $\alpha, \beta \in \mathbb{K}$, where the 2-cocycle $\varpi_{2}: \mathcal{L}_{\widetilde{\mathbb{A}}} \times \mathcal{L}_{\widetilde{\mathbb{A}}} \rightarrow \mathbb{K}$ is a skew-symmetric bilinear form satisfying the Jacobi identity:

$$
\varpi_{2}\left([a, b]_{D}, c\right)+\varpi_{2}\left([b, c]_{D}, a\right)+\varpi_{2}\left([c, a]_{D}, b\right)=0
$$

for any $a, b$ and $c \in \mathcal{L}_{\widetilde{\mathbb{A}}}$. It is evident that the existence of nontrivial central extensions on the Lie algebra $\mathcal{L}_{\widetilde{\mathbb{A}}}$ strongly depends on the algebraic structure of the algebra $\mathbb{A}$ underlying the whole construction presented above. Yet there exist some general algebraic properties which allow to proceed further with success. For example, assume that a smooth mapping

$$
\mathrm{D}: \mathcal{L}_{\widetilde{\mathbb{A}}} \rightarrow \operatorname{End} \mathcal{L}_{\widetilde{\mathbb{A}}}
$$

defines for any $u \in \mathcal{L}_{\widetilde{\mathbb{A}}}^{*} \simeq \mathcal{L}_{\widetilde{\mathbb{A}}}$ a weak derivation of the Lie algebra $\mathcal{L}_{\widetilde{\mathbb{A}}}$, that is

$$
\left(c, \mathrm{D}_{u}[a, b]_{D}\right)=\left(c,\left[\mathrm{D}_{u} a, b\right]_{D}+\left[a, \mathrm{D}_{u} b\right]_{D}\right)
$$

for any $a, b$ and $c \in \mathcal{L}_{\widetilde{\mathbb{A}}}$. Then the following important proposition $[69,89]$ holds.

Proposition 3.2. Let a linear mapping $\mathrm{D}_{u}: \mathcal{L}_{\widetilde{\mathbb{A}}} \rightarrow \mathcal{L}_{\widetilde{\mathbb{A}}}$ be for a fixed $u \in \mathcal{L}_{\widetilde{\mathbb{A}}}^{*}$ a weak skew-symmetric derivation of the Lie algebra $\mathcal{L}_{\widetilde{\mathbb{A}}}$. Then the expression

$$
\varpi_{2}(a, b):=\left(a, \mathrm{D}_{u} b\right)
$$


for any $a, b \in \mathcal{L}_{\widetilde{\mathbb{A}}}$ and $u \in \mathcal{L}_{\widetilde{\mathbb{A}}}^{*} \simeq \mathcal{L}_{\widetilde{\mathbb{A}}}$ defines a nontrivial 2-cocycle on the Lie algebra $\mathcal{L}_{\widetilde{\mathbb{A}}}$.

A proof is given by means of direct checking the Jacobi identity (3.5) and is omitted.

Remark 3.3. The expression (3.7) when linear in the element $u \in \mathcal{L}_{\widetilde{\mathbb{A}}}^{*}$ can be, evidently, represented for any $a, b \in \widetilde{\mathbb{A}}$ in the following scalar form:

$$
\left(a, \mathrm{D}_{u} b\right)=\left(u, \vartheta^{*}(a \wedge b)\right),
$$

where $\vartheta^{*}: \widetilde{\mathbb{A}} \wedge \widetilde{\mathbb{A}} \rightarrow \widetilde{\mathbb{A}}$ is some bilinear skew-symmetric mapping.

As follows from the results of Section (2) the right-hand side of expression (3.8) allows the equivalent form

$$
\left(u, \vartheta^{*}(a \wedge b)\right)=\left(u,[a, b]_{\bar{D}}\right),
$$

where the bracket $[a, b]_{\bar{D}}$ defines for any $a, b \in \widetilde{\mathbb{A}}$ a new adjacent Lie algebra structure on the loop algebra $\widetilde{\mathbb{A}}$, a priori compatible with the basic Lie structure $[\cdot, \cdot]_{D}$. In the case when these Lie structures coincide, that is $[\cdot, \cdot]_{D}=[\cdot, \cdot]_{\bar{D}}$, the cocycle $(3.7)$ naturally determines on the phase space $\mathcal{F}_{\widetilde{\mathbb{A}}}(u)$ the equivalent to $(3.4)$ Poisson bracket

$$
\{f, g\}(u):=\left(\nabla f(u(x)), \mathrm{D}_{u} \nabla g(u(x))\right)
$$

for any $f, g \in \mathcal{F}_{\widetilde{\mathbb{A}}}(u)$. Moreover, as follows from (3.9) and the ad-invariance property $(3.1)$, the mapping $\mathrm{D}_{u}(\cdot)=-[u, \cdot]_{D}$ for any $u \in \mathcal{L}_{\widetilde{\mathbb{A}}}^{*}$ is automatically a derivation of the weak adjacent Lie algebra $\mathcal{L}_{\widetilde{\mathbb{A}}}$.

Example 3.4. As a natural example of the derivation $D_{u}: \mathcal{L}_{\widetilde{\mathbb{A}}} \rightarrow \mathcal{L}_{\widetilde{\mathbb{A}}}$ one can take the mapping

$$
\mathrm{D}_{u}:=\Delta_{1} u(x) D_{x}+D_{x} \Delta_{2} u(x),
$$

where for $u \in \mathcal{L}_{\widetilde{\mathbb{A}}}^{*}$ the expressions $\Delta_{j} u(x): \widetilde{\mathbb{A}} \rightarrow \widetilde{\mathbb{A}}^{*}, j=1,2$, denote the convolution operators of the co-multiplication $\Delta: \widetilde{\mathbb{A}}^{*} \rightarrow \widetilde{\mathbb{A}}^{*} \otimes \widetilde{\mathbb{A}}^{*}$ with respect to its first and second tensor components, respectively. In particular, we have

$$
(\Delta u, a \otimes b):=(u, a \circ b)=\sum_{s, i, j=1}^{N} \sigma_{i j}^{s} \int_{\mathbb{S}^{1}} u_{s}(x) a^{i}(x) b^{j}(x) d x
$$

for a fixed $u=\sum_{s=1}^{N} u_{s}(x) u^{j} \in \widetilde{\mathbb{A}}^{*} \simeq \widetilde{\mathbb{A}}$ and any

$$
a=\sum_{i=1}^{N} a^{i}(x) e_{i}, \quad b=\sum_{j=1}^{N} b^{j}(x) e_{j} \in \mathcal{L}_{\widetilde{\mathbb{A}}}
$$


as we have assumed by definition, that $\left(u^{i}, e_{j}\right):=\delta_{j}^{i}, i, j=\overline{1, N}$. If now the weak Lie algebra $\mathcal{L}_{\widetilde{\mathbb{A}}}$ is generated by the commutator Lie structure $(3.4)$, that is

$$
[a, b]_{D}=D_{x} a \circ b-D_{x} b \circ a
$$

for any $a, b \in \mathcal{L}_{\tilde{\mathbb{A}}}$, it easy to check that the mapping (3.11) is a skewsymmetric with respect to the bilinear form $(\cdot, \cdot)$ on $\widetilde{\mathbb{A}}$ weak derivation of the Lie algebra $\mathcal{L}_{\widetilde{\mathbb{A}}}$. Moreover, the related weak Lie algebraic structure $[\cdot, \cdot]_{D^{\prime}}$ on $\widetilde{\mathbb{A}}$, satisfying the condition $\left(u,[a, b]_{D^{\prime}}\right)=\left(a, \mathrm{D}_{u} b\right)$ for any $a, b \in \mathcal{L}_{\widetilde{\mathbb{A}}}$, coincides exactly with that (3.12).

There are also other strictly algebraic tools for constructing Poisson brackets on the functional space $\mathcal{F}_{\widetilde{\mathbb{A}}}(u)$. For instance, as a simple consequence of Proposition 3.2 the following result [34, 69, 89] holds.

Proposition 3.5. Suppose that a nondegenerate linear skew-symmetric $\mathcal{R}$-matrix endomorphism $\mathcal{R}: \mathcal{L}_{\widetilde{\mathbb{A}}} \rightarrow \mathcal{L}_{\widetilde{\mathbb{A}}}$ satisfies the well-known weak YangBaxter commutator condition:

$$
\left(c,[\mathcal{R} a, \mathcal{R} b]_{D}\right)=\left(c, \mathcal{R}\left([\mathcal{R} a, b]_{D}+[a, \mathcal{R} b]_{D}\right)\right)=0
$$

for any $a, b$ and $c \in \mathcal{L}_{\widetilde{\mathbb{A}}}$. Then the inverse mapping $\mathcal{R}^{-1}: \mathcal{L}_{\widetilde{\mathbb{A}}} \rightarrow \mathcal{L}_{\widetilde{\mathbb{A}}}$ is a weak skew-symmetric derivation of the Lie algebra $\mathcal{L}_{\widetilde{\mathbb{A}}}$ and the expression

$$
\varpi_{2}(a, b)=\left(a, \mathcal{R}^{-1} b\right)
$$

defines for any $a, b \in \mathcal{L}_{\widetilde{\mathbb{A}}}$ a 2-cocycle on $\mathcal{L}_{\widetilde{\mathbb{A}}}$.

For any function $f \in \mathcal{F}_{\widetilde{\mathbb{A}}}(u)$ consider its differential $\delta f \in \Lambda^{1}(\widetilde{\mathbb{A}}(u))$ and define for a chosen element $h \in \widetilde{\mathbb{A}}(u)^{*}$ the vector field $\xi_{h} \in \Gamma(\widetilde{\mathbb{A}}(u))$ via the equality

$$
\delta f\left(\xi_{h}\right)=(\nabla f(u), h) .
$$

As symplectic forms on the phase space $\widetilde{\mathbb{A}}(u)$ are dual objects to the Poisson brackets on the functional space $\mathcal{F}_{\widetilde{\mathbb{A}}}(u)$, one easily obtains the following [69, 85, 89] proposition.

Proposition 3.6. Assume that the Lie algebra $\mathcal{L}_{\widetilde{\mathbb{A}}}$ allows a skew-symmetric nondegenerate $\mathcal{R}$-structure homomorphism $\mathcal{R}: \mathcal{L}_{\widetilde{\mathbb{A}}} \rightarrow \mathcal{L}_{\widetilde{\mathbb{A}}}$, satisfying the weak Yang-Baxter condition

$$
\left(c, \mathcal{R}\left([\mathcal{R} a, b]_{D}+[a, \mathcal{R} b]_{D}\right)-[\mathcal{R} a, \mathcal{R} b]_{D}\right)=0
$$

for any $a, b$ and $c \in \mathcal{L}_{\widetilde{\mathbb{A}}}$. Then differential 2 -forms

$$
\omega_{j}^{(2)} \in \Lambda^{2}(\widetilde{\mathbb{A}}(u)), \quad j=1,2,
$$


defined on the ideal $\widetilde{\mathbb{A}}(u) \subset \widetilde{\mathbb{A}}$ by

$$
\omega_{1}^{(2)}\left(\xi_{f}, \xi_{g}\right)=\left(\mathcal{R} \xi_{f}, \xi_{g}\right):=-\left(\vartheta_{1} \nabla f(u), \nabla g(u)\right),
$$

where

$$
\xi_{f}:=\vartheta_{1} \nabla f(u), \xi_{g}:=\vartheta_{1} \nabla g(u) \in \Lambda^{1}(\widetilde{\mathbb{A}}(u))
$$

with $f, g \in \mathcal{F}_{\widetilde{\mathbb{A}}}(u)$, and

$$
\omega_{2}^{(2)}\left(\xi_{f}, \xi_{g}\right)=\left(u,\left[\mathcal{R} \xi_{f}, \mathcal{R} \xi_{f}\right]_{D}\right):=-\left(\vartheta_{2} \nabla f(u), \nabla g(u)\right),
$$

where

$$
\xi_{f}:=\vartheta_{2} \nabla f(u), \xi_{g}:=\vartheta_{2} \nabla g(u) \in \Lambda^{1}(\widetilde{\mathbb{A}}(u))
$$

with $f, g \in \mathcal{F}_{\widetilde{\mathbb{A}}}(u)$, are closed. Moreover, the corresponding Hamiltonian operators

$$
\vartheta_{1}, \vartheta_{2}: \mathcal{L}_{\widetilde{\mathbb{A}}} \rightarrow \mathcal{L}_{\widetilde{\mathbb{A}}}^{*}
$$

are compatible, that is for arbitrary $\lambda, \mu \in \mathbb{K}$ the sum $\lambda \vartheta_{1}+\mu \vartheta_{2}: \mathcal{L}_{\widetilde{\mathbb{A}}} \rightarrow \mathcal{L}_{\widetilde{\mathbb{A}}}$ is also a Hamiltonian operator.

Proof. (Sketch). The 2-form (3.16) is closed, as the expression (3.16) determines a 2-cocycle on the Lie algebra $\mathcal{L}_{\widetilde{\mathbb{A}}}$ owing to the fact that the inverse mapping $\mathcal{R}^{-1}: \mathcal{L}_{\widetilde{\mathbb{A}}} \rightarrow \mathcal{L}_{\widetilde{\mathbb{A}}}$ is a weak derivation on $\mathcal{L}_{\widetilde{\mathbb{A}}}$, that is

$$
\left(c, \mathcal{R}^{-1}[a, b]_{D}\right)=\left(c,\left[\mathcal{R}^{-1} a, b\right]_{D}+\left[a, \mathcal{R}^{-1} b\right]_{D}\right)
$$

for any $a, b$ and $c \in \mathcal{L}_{\widetilde{\mathbb{A}}}$. A proof of the second part of the proposition consists in direct checking the closedness of the 2-forms $\omega_{2}^{(2)} \in \Lambda^{2}(\widetilde{\mathbb{A}}(u))$, which is equivalent to the Yang-Baxter condition (3.15).

Concerning their compatibility, we observe that the Hamiltonian operator $\vartheta_{2}: \mathcal{L}_{\widetilde{\mathbb{A}}} \rightarrow \mathcal{L}_{\widetilde{\mathbb{A}}}$, corresponding to the expression (3.17), is representable as the composition $\vartheta_{2}=\vartheta_{1}\left(\vartheta_{0}^{-1} \vartheta_{1}\right)$, where the Hamiltonian operator $\vartheta_{0}: \mathcal{L}_{\widetilde{\mathbb{A}}} \rightarrow \mathcal{L}_{\widetilde{\mathbb{A}}}^{*}$ is naturally determined from the canonical Lie-Poisson bracket (3.4) as

$$
(u,[\nabla f(u), \nabla g(u)]):=\left(\vartheta_{0} \nabla f(u), \nabla g(u)\right)
$$

for any $f, g \in \mathcal{F}_{\widetilde{\mathbb{A}}}(u)$. From this representation one easily derives $[22,20$, $69,43,89]$ the compatibility of the Hamiltonian operators $\vartheta_{2}$ and $\vartheta_{1}$ on $\widetilde{\mathbb{A}}(u)$, following from the evident compatibility of operators $\vartheta_{0}$ and $\vartheta_{1}$ on $\widetilde{\mathbb{A}}(u)$, owing to 2-cocycle property of the bilinear form (3.14).

The $\mathcal{R}$-structures on the weak Lie algebra $\mathcal{L}_{\widetilde{\mathbb{A}}}$ can be effectively exploited for constructing additional Hamiltonian operators on $\mathcal{L}_{\widetilde{\mathbb{A}}}^{*}$ owing to the fact that the bracket

$$
\left(c,[a, b]_{(\mathcal{R})}\right):=\left(c,[a, \mathcal{R} b]_{D}+[\mathcal{R} a, b]_{D}\right)
$$


generates for any $a, b, c \in \mathcal{L}_{\widetilde{\mathbb{A}}}$ a new weak Lie structure on the linear space $\mathcal{L}_{\widetilde{\mathbb{A}}}$, thus producing a new weak Lie algebra $\mathcal{L}_{\widetilde{\mathbb{A}}}^{(\mathcal{R})}:=\left(\widetilde{\mathbb{A}},[\cdot, \cdot]_{(\mathcal{R})}\right)$.

Example 3.7. Consider the Rota-Baxter [86, 87] endomorphism

$$
\mathcal{R}: \mathcal{L}_{\widetilde{\mathbb{A}}} \rightarrow \mathcal{L}_{\widetilde{\mathbb{A}}}
$$

where for any $a \in \mathcal{L}_{\widetilde{\mathbb{A}}}$ we put

$$
\mathcal{R}(a):=\frac{1}{2}\left[\int_{0}^{x} a(y) d y-\int_{x}^{2 \pi} a(y) d y\right]
$$

for any $x \in \mathbb{S}^{1}$, which satisfies the weak Yang-Baxter commutator condition (3.13). Then it is easy to check that the inverse mapping $\mathcal{R}^{-1}=d / d x$, $x \in \mathbb{S}^{1}$, is the natural skew-symmetric derivation of the weak Lie algebra $\mathcal{L}_{\widetilde{\mathbb{A}}}$, generating a Poisson structure compatible with that of (3.10).

In a manner similar to the above $[69,57,85,89]$ one verifies the existence of the following so called "quadratic" Poisson brackets. Namely, the next proposition holds.

Proposition 3.8. Suppose that the weak Yang-Baxter condition (3.15) hold. Then the brackets

$$
\{f, g\}_{1}:=(u \circ \nabla f(u), \mathcal{R}(u \circ \nabla g(u)))-(\nabla f(u) \circ u, \mathcal{R}(\nabla g(u) \circ u))
$$

and

$$
\{f, g\}_{2}:=\left(u,[\mathcal{R} \nabla f(u), \nabla g(u)]_{D}+[\nabla f(u), \mathcal{R} \nabla g(u)]_{D}\right)
$$

defined for any $f, g \in \mathcal{F}_{\widetilde{\mathbb{A}}}(u)$, are Poisson and compatible on $\widetilde{\mathbb{A}}(u)$.

As an interesting and also useful consequence of the $\mathcal{R}$-matrix construction, one has the fact that the following subspaces

$$
\mathcal{L} \frac{ \pm}{\widetilde{\mathbb{A}}}:=(\mathbb{I} \pm \mathcal{R}) / 2 \in \mathcal{L}_{\widetilde{\mathbb{A}}}
$$

are Lie subalgebras of $\mathcal{L}_{\widetilde{\mathbb{A}}}$, which is equivalent to the condition that the mappings

$$
(\mathbb{I} \pm \mathcal{R}) / 2: \mathcal{L}_{\widetilde{\mathbb{A}}}^{(\mathcal{R})} \rightarrow \mathcal{L}_{\widetilde{\mathbb{A}}}^{ \pm} \subset \mathcal{L}_{\widetilde{\mathbb{A}}}
$$

are homomorphisms [89] of the Lie algebras $\mathcal{L}_{\widetilde{\mathbb{A}}}^{(\mathcal{R})}:=\left(\widetilde{\mathbb{A}},[\cdot, \cdot]_{(\mathcal{R})}\right)$ and $\mathcal{L}_{\widetilde{\mathbb{A}}}^{ \pm}$. In the special case when $\mathcal{L}_{\widetilde{\mathbb{A}}}^{+} \cap \mathcal{L}_{\widetilde{\mathbb{A}}}^{-}=\{0\}$, the operator $\mathcal{R}=P_{+}-P_{-}$and the linear operators $P_{ \pm}:=(\mathbb{I} \pm \mathcal{R}) / 2: \mathcal{L}_{\widetilde{\mathbb{A}}} \rightarrow \mathcal{L}_{\widetilde{\mathbb{A}}}$ are projectors and the weak Lie algebra $\mathcal{L}_{\widetilde{\mathbb{A}}}$ allows the direct sum splitting $\mathcal{L}_{\widetilde{\mathbb{A}}}=\mathcal{L}_{\widetilde{\mathbb{A}}}^{+} \oplus \mathcal{L}_{\widetilde{\mathbb{A}}}^{-}$. 


\section{Integrable Riemann hydRodynamical Systems And RELATED MULTICOMPONENT HAMILTONIAN OPERATORS}

4.1. General setting. In preceding sections we have shown that any skewsymmetric Lie structure $[\cdot, \cdot]_{D}$ on the weak adjacent Lie algebra $\mathcal{L}_{\widetilde{\mathbb{A}}}$, satisfying the Jacobi identity and nontrivially depending on the derivation $D_{x}: \widetilde{\mathbb{A}} \rightarrow \widetilde{\mathbb{A}}$, determines on the phase space $\mathcal{F}_{\widetilde{\mathbb{A}}}(u)$ the local Poisson bracket

$$
\{f, g\}(u):=\left(u,[\nabla f(u(x)), \nabla g(u(x))]_{D}\right)
$$

for all $u \in \widetilde{\mathbb{A}}^{*}$ and arbitrary functions $f, g \in \mathcal{F}_{\widetilde{\mathbb{A}}}(u)$. Moreover, from the analysis provided above we know that if the Hamiltonian operator $\vartheta(u): \mathcal{L}_{\widetilde{\mathbb{A}}} \rightarrow \mathcal{L}_{\widetilde{\mathbb{A}}}^{*} \simeq \mathcal{L}_{\widetilde{\mathbb{A}}}$ related (2.29) corresponds to some 2-cocycle on the Lie algebra $\mathcal{L}_{\widetilde{\mathbb{A}}}$, then it will be a priori Hamiltonian. Moreover, owing to Proposition 3.2, if this 2-cocycle is generated by a derivation $\mathrm{D}_{u}: \mathcal{L}_{\widetilde{\mathbb{A}}} \rightarrow \mathcal{L}_{\widetilde{\mathbb{A}}}$, $u \in \widetilde{\mathbb{A}}^{*} \simeq \widetilde{\mathbb{A}}$, on the Lie algebra $\mathcal{L}_{\widetilde{\mathbb{A}}}$, one need only check the related weak Leibniz property $(3.6)$ in the weak adjacent Lie algebra $\mathcal{L}_{\widetilde{\mathbb{A}}}$. In Section 2 we already showed that the skew-symmetric structure

$$
[a, b]_{D}=D_{x} a \circ b-D_{x} b \circ a
$$

for any $a, b \in \mathcal{L}_{\widetilde{\mathbb{A}}}$, imposed on the weak adjacent Lie algebra $\mathcal{L}_{\widetilde{\mathbb{A}}}$, gives rise to the Hamiltonian operator $(2.30)$ on $\widetilde{\mathbb{A}}(u)$

$$
\vartheta(u)=\sigma(u) D_{x}+D_{x} \sigma(u)^{\top}
$$

and to the related multiplicative Balinsky-Novikov algebra structure (2.32) on $\mathbb{A}$

$$
\left[R_{a}, R_{b}\right]=0,\left[L_{a}, L_{b}\right]=L_{[a, b]}
$$

for any $a, b$ and $c \in \mathbb{A}$. Similarly, the skew-symmetric structure (2.34)

$$
[a, b]_{D}=D_{x}^{-1} a \circ b-D_{x}^{-1} b \circ a
$$

for any $a, b \in \mathcal{L}_{\widetilde{\mathbb{A}}}$ on the weak adjacent Lie algebra $\mathcal{L}_{\widetilde{\mathbb{A}}}$ gives rise to the Hamiltonian operator $(2.30)$ on $\widetilde{\mathbb{A}}(u)$

$$
\vartheta(u)=\sigma(u) D_{x}^{-1}+D_{x}^{-1} \sigma(u)^{\top}
$$

and to the related multiplicative right Leibniz algebra structure (2.32) on $\mathbb{A}$

$$
R_{a \circ b}=\left[R_{a}, R_{b}\right], \quad \quad R_{a \circ b}+R_{b \circ a}=0 .
$$

Moreover, the skew-symmetric structure (2.34)

$$
[a, b]_{D}=D_{x}^{-1} b \circ D_{x} a-D_{x}^{-1} a \circ D_{x} b
$$


for any $a, b \in \mathcal{L}_{\widetilde{\mathbb{A}}}$ on the weak adjacent Lie algebra $\mathcal{L}_{\widetilde{\mathbb{A}}}$ gives rise to the Hamiltonian operator $(2.38)$ on $\widetilde{\mathbb{A}}(u)$

$$
\vartheta(u)=D_{x} \sigma(u) D_{x}^{-1}-D_{x}^{-1} \sigma(u)^{\top} D_{x}
$$

and to the related multiplicative Riemann algebra structure (2.39) on $\mathbb{A}$

$$
\left[R_{a}, R_{b}\right]=0, \quad L_{a \circ b}=R_{a \circ b}=L_{b \circ a}
$$

for all $a, b, c \in \mathbb{A}$.

Remark 4.2. Just as in Section 2, one can construct for all $a, b, c \in \mathbb{A}$ dual Balinsky-Novikov

$$
R_{[a, b]}=\left[R_{b}, R_{a}\right], \quad\left[L_{a}, L_{b}\right]=0,
$$

Leibniz

$$
L_{a \circ b}=\left[L_{a}, L_{b}\right], \quad L_{a \circ b}+L_{b \circ a}=0
$$

and Riemann

$$
\left[L_{a}, L_{b}\right]=0, \quad R_{a \circ b}=L_{a \circ b}=R_{b \circ a}
$$

algebra constraints, respectively related to the adjacent Lie algebra $\mathcal{L}_{\widetilde{\mathbb{A}}}$ structures

$$
\begin{gathered}
{[a, b]_{D}=D_{x} a \circ b-D_{x} b \circ a,} \\
{[a, b]_{D}=a \circ D_{x}^{-1} b-b \circ D_{x}^{-1} a,} \\
{[a, b]_{D}=-D_{x} a \circ D_{x}^{-1} b+D_{x} b \circ D_{x}^{-1} a}
\end{gathered}
$$

for any $a, b \in \mathcal{L}_{\widetilde{\mathbb{A}}}$.

As mentioned above, simultaneously we have shown that the expressions (4.1), (4.3) and (4.4) are true Hamiltonian operators on the functional space $\mathcal{F}_{\widetilde{\AA}}(u)$ satisfying the Schouten-Nijenhuis condition (2.10). Using the algebraic scheme in [91] and the right Leibniz algebra (4.3) and the new Riemann algebra (4.4), one can describe a wide class of multicomponent completely integrable dynamical systems containing, as follows from the recent results in [80], infinite hierarchies of the multicomponent hydrodynamical Riemann type systems.

As the expressions (2.30), (2.36) and (2.40) are true Hamiltonian operators on the functional space $\mathcal{F}_{\widetilde{\mathbb{A}}}(u)$ satisfying the Schouten-Nijenhuis condition (2.10), following the algebraic scheme of [91] mentioned above and using the results of [80] and the right Leibniz algebra (2.35) and the new Riemann algebra (2.39), one can describe a wide class of multicomponent completely integrable dynamical systems containing the infinite hierarchies 
of multicomponent Riemann hydrodynamical flows. For instance, consider the generalized completely integrable Riemann type dynamical system

$$
D_{t} u_{1}=u_{2}, D_{t} u_{2}=u_{3}, \ldots, D_{t} u_{N}=0
$$

on the functional space $\widetilde{\mathbb{A}}(u)$ for some nonassociative and noncommutative finite-dimensional algebra $\mathbb{A}$, where

$$
D_{t}:=\partial / \partial t+u_{1} D_{x}, \quad D_{x}:=\partial / \partial x,
$$

$x \in \mathbb{S}^{1}, N \in \mathbb{Z}_{+}$, which was recently studied in detail in $[77,80]$. The relationships (2.39) allow to calculate the corresponding representations of the Riemann algebra $\mathbb{A}$ for cases $N=2$ and $N=3$, giving rise to the corresponding Hamiltonian operators $\vartheta(u): \mathcal{L}_{\widetilde{\mathbb{A}}} \rightarrow \mathcal{L}_{\widetilde{\mathbb{A}}}$, coinciding with those constructed in [80] modulo the trivial constant 2-cocycles on the weak adjacent loop Lie algebra $\mathcal{L}_{\widetilde{\mathbb{A}}}$. In fact, for the case $N=2$ one easily obtains from (2.40) the skew-symmetric two-dimensional matrix derivation representation

$$
\vartheta_{2}(u):=\left(\begin{array}{cc}
0 & u_{1, x} D_{x}^{-1} \\
D_{x}^{-1} u_{1, x} & u_{2, x} D_{x}^{-1}+D_{x}^{-1} u_{2, x}
\end{array}\right),
$$

coinciding, modulo the trivial constant 2-cocycle $\varpi_{2}(a, b):=f_{2}\left(D_{x}^{-1} a, b\right)$, determined for a suitable symmetric bilinear form $f_{2}: \mathcal{L}_{\widetilde{\mathbb{A}}} \times \mathcal{L}_{\widetilde{\mathbb{A}}} \rightarrow \mathbb{K}$ and all $a, b \in \mathcal{L}_{\widetilde{\mathbb{A}}}$, with the Hamiltonian operator

$$
\eta_{2}(u)=\left(\begin{array}{cc}
D_{x}^{-1} & u_{1, x} D_{x}^{-1} \\
D_{x}^{-1} u_{1, x} & u_{2, x} D_{x}^{-1}+D_{x}^{-1} u_{2, x}
\end{array}\right),
$$

on the space $\mathcal{F}_{\widetilde{\mathbb{A}}}(u)$ for the Riemann type dynamical system (4.6), whose Hamiltonian representation

$$
\frac{d}{d t}\left(u_{1}, u_{2}\right)^{\top}=-\eta_{2}(u) \nabla H_{2}\left(u_{1}, u_{2}\right)
$$

holds for the Hamiltonian function $H_{2} \in \mathcal{F}_{\widetilde{\mathbb{A}}}(u)$, equal to

$$
H_{2}:=\frac{1}{2} \int_{0}^{2 \pi}\left(u_{2} u_{1, x}-u_{1} u_{2, x}\right) d x .
$$

Proceeding similarly for the case $N=3$, one easily obtains from (2.40) the skew-symmetric three-dimensional matrix Hamiltonian operator

$$
\vartheta_{3}(u): \mathcal{L}_{\widetilde{\mathbb{A}}} \rightarrow \mathcal{L}_{\widetilde{\mathbb{A}}}
$$

representation

$$
\vartheta_{3}(u)=\left(\begin{array}{ccc}
0 & u_{1, x} D_{x}^{-1} & 0 \\
D_{x}^{-1} u_{1, x} & u_{2, x} D_{x}^{-1}+D_{x}^{-1} u_{2, x} & D_{x}^{-1} u_{3, x} \\
0 & u_{3, x} D_{x}^{-1} & 0
\end{array}\right)
$$


coinciding, modulo the trivial constant 2-cocycle

$$
\varpi_{2}(a, b):=f_{3}\left(D_{x}^{-1} a, b\right),
$$

determined for a suitable symmetric bilinear form $f_{3}: \widetilde{\mathbb{A}} \times \widetilde{\mathbb{A}} \rightarrow \mathbb{K}$ and all $a, b \in \mathcal{L}_{\widetilde{\mathbb{A}}}$ with the Hamiltonian operator

$$
\eta_{3}(u)=\left(\begin{array}{ccc}
D_{x}^{-1} & u_{1, x} D_{x}^{-1} & 0 \\
D_{x}^{-1} u_{1, x} & u_{2, x} D_{x}^{-1}+D_{x}^{-1} u_{2, x} & D_{x}^{-1} u_{3, x} \\
0 & u_{3, x} D_{x}^{-1} & 0
\end{array}\right)
$$

on the functional space $\mathcal{F}_{\widetilde{\mathbb{A}}}(u)$ for the Riemann dynamical system (4.6), whose Hamiltonian representation

$$
\frac{d}{d t}\left(u_{1}, u_{2}, u_{3}\right)^{\top}=-\eta_{3}(u) \nabla H_{3}\left[u_{1}, u_{2}, u_{3}\right]
$$

holds for a suitably constructed Hamiltonian function $H_{3} \in \mathcal{F}_{\widetilde{\mathbb{A}}}(u)$.

There is also an interesting observation concerning an infinite hierarchy [80] of the generalized Riemann hydrodynamic systems

$$
D_{t} u_{1}=u_{2}, \quad D_{t} u_{2}=u_{3}, \quad \ldots, \quad D_{t} u_{N-1}=\left(\bar{u}_{N, x}\right)^{s}, \quad D_{t} \bar{u}_{N}=0
$$

on the functional space $\mathcal{F}_{\widetilde{\mathbb{A}}}(u)$, where $s, N \in \mathbb{Z}_{+}$, with the algebra $\mathbb{A}$ generated by the constraints (2.39). For the case $s=2$ and $N=3$ the above skew-symmetric three-dimensional matrix Hamiltonian operator $\bar{\vartheta}_{3 \mid 2}(u): \mathcal{L}_{\widetilde{\mathbb{A}}} \rightarrow \mathcal{L}_{\widetilde{\mathbb{A}}}$ representation

$$
\bar{\vartheta}_{3 \mid 2}(u)=\left(\begin{array}{ccc}
0 & u_{1, x} D_{x}^{-1} & 0 \\
D_{x}^{-1} u_{1, x} & u_{2, x} D_{x}^{-1}+D_{x}^{-1} u_{2, x} & D_{x}^{-1} \bar{u}_{3, x} \\
0 & \bar{u}_{3, x} D_{x}^{-1} & 0
\end{array}\right)
$$

proves to coincide, modulo the trivial constant 2-cocycle

$$
\bar{\omega}_{2, \bar{\eta}}(a, b):=f_{\bar{\eta}}\left(D_{x}^{-1} a, b\right),
$$

determined for any suitable symmetric bilinear form $f_{\bar{\eta}}: \mathcal{L}_{\widetilde{\mathbb{A}}} \times \mathcal{L}_{\widetilde{\mathbb{A}}} \rightarrow \mathbb{K}$ and all $a, b \in \mathcal{L}_{\widetilde{\mathbb{A}}}$ exactly with the Hamiltonian operator

$$
\bar{\eta}_{3 \mid 2}(u)=\left(\begin{array}{ccc}
D_{x}^{-1} & u_{1, x} D_{x}^{-1} & 0 \\
D_{x}^{-1} u_{1, x} & u_{2, x} D_{x}^{-1}+D_{x}^{-1} u_{2, x} & D_{x}^{-1} \bar{u}_{3, x} \\
0 & \bar{u}_{3, x} D_{x}^{-1} & 0
\end{array}\right)
$$

on the functional space $\mathcal{F}_{\widetilde{\mathbb{A}}}(u)$ for the Riemann type dynamical system (4.7), whose Hamiltonian representation

$$
\frac{d}{d t}\left(u_{1}, u_{2}, \bar{u}_{3}\right)^{\top}=-\bar{\eta}_{3 \mid 2}(u) \nabla H_{3 \mid 2}\left(u_{1}, u_{2}, \bar{u}_{3}\right)
$$


holds for the Hamiltonian function $H_{3 \mid 2} \in \mathcal{F}_{\widetilde{\mathbb{A}}}(u)$, equal to

$$
H_{3 \mid 2}:=\frac{1}{2} \int_{0}^{2 \pi}\left[2 u_{1}\left(\bar{u}_{3, x}\right)^{2}-u_{2}^{2}-u_{1}^{2} u_{2, x}\right] d x .
$$

Moreover, one can calculate such a constant 2-cocycle on the Lie algebra $\mathcal{L}_{\widetilde{\mathbb{A}}}$

$$
\varpi_{2, \bar{\vartheta}}(a, b):=f_{\bar{\vartheta}}^{(1)}\left(D_{x}^{-1} a, b\right)+f_{\bar{\vartheta}}^{(2)}(a, b),
$$

determined for any $a, b \in \mathcal{L}_{\widetilde{\mathbb{A}}}$ by means of two suitably symmetric

$$
f_{\bar{\vartheta}}^{(1)}: \mathcal{L}_{\widetilde{\mathbb{A}}} \times \mathcal{L}_{\widetilde{\mathbb{A}}} \rightarrow \mathbb{K}
$$

and skew-symmetric

$$
f_{\bar{\vartheta}}^{(2)}: \mathcal{L}_{\widetilde{\mathbb{A}}} \times \mathcal{L}_{\widetilde{\mathbb{A}}} \rightarrow \mathbb{K}
$$

bilinear forms, which naturally generates the (4.8)-compatible Hamiltonian operator

$$
\bar{\vartheta}_{3 \mid 2}^{(0)}(u)=\left(\begin{array}{ccc}
0 & 1 & 0 \\
-1 & 0 & 0 \\
0 & 0 & 1 / 2 D_{x}^{-1}
\end{array}\right)
$$

on the functional space $\mathcal{F}_{\widetilde{\mathbb{A}}}(u)$ for $(4.7)$, whose Hamiltonian representation

$$
\frac{d}{d t}\left(u_{1}, u_{2}, \bar{u}_{3}\right)^{\top}=-\bar{\vartheta}_{3 \mid 2}^{(0)}(u) \nabla H_{3 \mid 2}^{(0)}\left(u_{1}, u_{2}, \bar{u}_{3}\right)
$$

holds for the Hamiltonian function $H_{3 \mid 2}^{(0)} \in \mathcal{F}_{\widetilde{\mathbb{A}}}(u)$, equal to

$$
H_{3 \mid 2}^{(0)}:=\frac{1}{2} \int_{0}^{2 \pi}\left[u_{1} u_{2, x}-u_{2} u_{1, x}-2\left(\bar{u}_{3, x}\right)^{2}\right] d x .
$$

It is worth noting here, as was already remarked in [81], that the generalized Riemann hydrodynamic system (4.7) for $s=3, N=3$ reduces to the well-known integrable Degasperis-Processi dynamical system [32, 31] for the function $u:=u_{1}$ :

$$
u_{t}-u_{x x t}+4 u u_{x}-3 u_{x} u_{x x}-u u_{x x x}=0 .
$$

Also, for $s=2$ and $N=3$, the system (4.7) for the function $u:=u_{1}$ reduces to the well-known [29] integrable Camassa-Holm dynamical system

$$
u_{t}-u_{x x t}+3 u u_{x}-2 u_{x} u_{x x}+u u_{x x x}=0,
$$

whose multicomponent extensions were recently extensively studied in [39, $46,30,91]$.

Now, returning to the case $N=2$ of the system (4.6), it reduces under the substitutions $u_{1}:=u, u_{2}:=D_{x}^{-1} u_{x}^{2} / 2$ to the well-known $[47,44,74,79]$ Hunter-Saxton nonlinear dynamical system

$$
d u / d t=-u u_{x}+D_{x}^{-1} u_{x}^{2} / 2
$$


on the functional manifold $\widetilde{\mathbb{A}}(u), u \in \widetilde{\mathbb{A}}^{*}$, describing propagation of shortwaves in a relaxing medium with spatial memory effects. As shown in [74, $79,75]$, the dynamical system (4.9) is a completely integrable bi-Hamiltonian flow on the functional manifold $\widetilde{\mathbb{A}}(u), u \in \widetilde{\mathbb{A}}^{*}$, with respect to the compatible pair of scalar Hamiltonian operators

$$
\begin{array}{rlrl}
\vartheta_{1}(u), \vartheta_{2}(u): T^{*}(\widetilde{\mathbb{A}}(u)) & \rightarrow T(\widetilde{\mathbb{A}}(u)), \\
\vartheta_{1}(u)=D_{x}^{-1}, & \vartheta_{2}(u) & =u D_{x}^{-1}+D_{x}^{-1} u .
\end{array}
$$

As we are interested in the corresponding multicomponent generalization of the dynamical system (4.9), we need to consider the functional space $\widetilde{\mathbb{A}}(u)$, $u \in \widetilde{\mathbb{A}}^{*}$, generated by a finite-dimensional noncommutive and nonassociative algebra $\mathbb{A}$, and construct the Poisson operators on $\mathcal{F}_{\widetilde{\mathbb{A}}}(u)$ in the form $(2.36)$, related to the right Leibniz algebra structure (2.35) and reducing at $N=1$ to the scalar Hamiltonian operator $\vartheta_{2}(u): T^{*}(\widetilde{\mathbb{A}}(u)) \rightarrow T(\widetilde{\mathbb{A}}(u))$ from the pair (4.10).

Moreover, as the compatible Hamiltonian operators are generated by means of suitable central extentions of the adjacent weak Lie algebra, the problem of description them, as was noted above, requires a detailed investigation of the structural properties and finite-dimensional representations of the right Leibniz algebras defined by the constraints (2.35). In what will follow, we stop mostly on the structural properties of the right Leibniz algebras, defined by the constraints (2.35), in particular, we characterize in detail the related derivation algebras.

\section{Preliminary algebraic setting}

An algebra $(L,+, \cdot)$ over a field $\mathbb{K}$ is called a (right) Leibniz algebra if it satisfies the identity

$$
x(y z)=(x y) z-(x z) y
$$

for any $x, y, z \in L$. Any Lie algebra is clearly a Leibniz algebra.

Leibniz algebras were introduced by A.M. Bloh [23, 24] and rediscovered by J.-L. Loday [59]. Recall that a subalgebra $H \subseteq L$ is said to be an ideal of a Leibniz algebra $L$ if $H \cdot L, L \cdot H \subseteq H$. A linear mapping $\delta: L \rightarrow L$ is called a derivation of $L$ if

$$
\delta(x y)=\delta(x) y+x \delta(y)
$$

for all $x, y \in L$. The set Der $L$ of all derivations of a Leibniz algebra $L$ is a Lie algebra due to operations of the addition "+" and the commutation " $[-,-]$ " of linear operators in $L$. The operator

$$
r_{a}: L \ni x \mapsto x a \in L
$$


of a right multiplication is a derivation of $L$ (so-called an inner derivation of $L$ induced by $a \in L)$. The set

$$
\operatorname{Inn} L:=\left\{r_{a} \mid a \in L\right\}
$$

of all inner derivations of $L$ is an ideal of the Lie $\operatorname{ring} \operatorname{Der} L$ (see e.g. [41]. A general theory for inner derivations in nonassociative algebras is given in [88]. If $L$ is a Leibniz algebra, then

$$
\operatorname{Leib}(L):=\operatorname{spa}\left\{x^{2} \mid x \in L\right\}
$$

is the smallest ideal of $L$ such that the quotient algebra $L / \operatorname{Leib}(L)$ is a Lie algebra (see e.g. [15, 33]. The center

$$
Z(L):=\{z \in L \mid z L=0=L z\}
$$

and the right annihilator

$$
\operatorname{rann} L:=\{t \in L \mid L t=0\}
$$

of $L$ are ideals in $L$ such that $Z(L)$, Leib $(L) \subseteq \operatorname{rann} L$.

A linear mapping $F: L \rightarrow L$ is called a generalized derivation of a Leibniz algebra $L$ associated with a derivation $\delta \in \operatorname{Der} L$ (in the sense of Brežar [26] if

$$
F(x y)=F(x) y+x \delta(y)
$$

for all $x, y \in L$. Denote by GDer $L$ the set of all generalized derivations of $L$. We will write $(F, \delta) \in \mathrm{GDer} L$ if and only if $F$ is a generalized derivation of $L$ associated with $\delta \in \operatorname{Der} L$. Since $(\delta, \delta) \in \operatorname{GDer} L$ for any $\delta \in \operatorname{Der} L$, one concludes that

$$
\operatorname{Inn} L \subseteq \operatorname{Der} L \subseteq \operatorname{GDer} L .
$$

A generalized derivation $F$ of $L$ that is associated with an inner derivation $r_{a} \in \operatorname{Inn} L$ is called a generalized inner derivation of $L$. By IGDer $L$ we denote the set of all generalized inner derivations of $L$. Another various generalizations of Lie (and Leibniz) algebra derivations was introduced in $[15,28,42,56,63]$ and others.

In what follows, let $D=\operatorname{Der} L, G=\operatorname{GDer} L, \Delta$ be a nonempty subset of $D$ (respectively $G$ ). If $I$ is an ideal of $L$ and $\delta(I) \subseteq I$ for all $\delta \in \Delta$, then $I$ is called a $\Delta$-ideal of $L$. Inasmuch $(x+d(x))(x+d(x)) \in \operatorname{Leib}(L)$ for any $x \in L$ and $d \in D$, we deduce that $d\left(x^{2}\right) \in \operatorname{Leib}(L)$ and so Leib $(L)$ is a $D$-ideal of $L$. For a Leibniz algebra $(L,+, \cdot)$, define the derived sequence as follows:

$$
L^{1}=L, \quad L^{2}=L L, \quad L^{(k+1)}=L^{(k)} L, \quad(k \geqslant 1) .
$$

A Leibniz algebra $L$ is called nilpotent if there exists a positive integer $s$ such that $L^{(s)}=0$ (see e.g. $\left.[2,33,42,16]\right)$. For a (Lie or Leibniz) algebra 
$L, X \in\{0, D, G\}$ and

$$
W= \begin{cases}0 & \text { if } Y=\operatorname{Leib}(L) \text { and } \bar{X}=X \in\{0, D\} \\ Y & \text { if } Y=\operatorname{rann} L \text { and } X=G\end{cases}
$$

the algebra $A / W$ is called:

- $\bar{X}$-semisimple if, for any $X$-ideal $T$ of $A$, the condition $T^{2} \subseteq Y$ implies that $T \subseteq Y$,

- $\bar{X}$-prime if, for any $X$-ideals $T, Q$ of $A$, the condition $T Q \subseteq Y$ implies that $T \subseteq Y$ or $Q \subseteq Y$,

- $\bar{X}$-simple if $[A, A] \neq Y$ and it only has the following $X$-ideals: $0, Y$ and $A$ (here 0 and $Y$ are not necessarily different),

- $\bar{X}$-primary if, for any $X$-ideals $T, Q$ of $A$, the condition $T Q \subseteq Y$ implies that $T \subseteq Y$ or $Q^{m} \subseteq Y$ for some positive integer $m$.

In particular, a $\bar{X}$-semisimple (respectively $\bar{X}$-prime, $\bar{X}$-simple or $\bar{X}$ primary) Lie (or Leibniz) algebra $L$ is called semisimple (respectively prime, simple or primary) if $\bar{X}=0$ and a 0 -ideal is an ideal of $L$. A Leibniz algebra $L$ is semisimple (respectively prime, simple or primary) if and only if the Lie algebra $L / \operatorname{Leib}(L)$ is the ones. If $L$ is a simple Leibniz algebra, then $L /$ Leib $(L)$ is a simple Lie algebra, but the opposite is not true. It is not difficult to check that if a Leibniz algebra $L$ is prime (respectively semisimple or simple), then $\operatorname{rann} L=\operatorname{Leib}(L)$. Every semisimple (respectively prime, simple or primary) Leibniz algebra is $D$-semisimple (respectively $D$-prime, $D$-simple or $D$-primary).

The Leibniz algebras are very popular in physics. Many authors have investigated derivations of Leibniz algebras in the context of geometric study of algebras (see e.g. $[54,72,83,82,84]$ ) and representations of Leibniz algebras (see e.g. [42, 41, 60, 63]). For example, A. Fialowski, A. Kh. Khudoyberdiyev and B. A. Omirov [42] have proved that a Leibniz algebra is nilpotent if and only if it admits an invertible Leibniz-derivation, B. A. Omirov [72], I. S. Rakhimov and A.-H. Al-Nashi [84] have studied derivation algebras of filiform Leibniz algebras, M. Ladra, I. M. Rikhsiboev and R. M. Turdibaev [54] have proved that a finite-dimensional Leibniz algebra $L$ with a nonsingular derivation is nilpotent, I. S. Rakhimov, K. K. Masutova and B. A. Omiro [83] have proved, in particular, that any derivation of a simple finite-dimensional Leibniz algebra over a field of zero characteristic can be represented as sum of three derivations of special form. In this paper we study connections between Leibniz algebras $L$, their derivation algebras Der $L$ and generalized derivation algebras GDer $L$.

Our first result subject to these topics is the following proposition which will be proved in Section 6: 
Proposition 5.1. Let $L$ be a Leibniz algebra. Then the following hold:

(1) $D$ is a simple Lie algebra if and only if $L$ is a simple Leibniz algebra and $D=\operatorname{Inn} L=[D, D]$,

(2) if $D$ is a prime (respectively semisimple or primary) Lie algebra, then $L$ is a D-prime (respectively D-semisimple or D-primary) Leibniz algebra.

We also prove an analogue of the result of S. Tôgô [93] that is a finitedimensional Leibniz algebra $L$ such that $L \neq L^{2}$ and $Z(L) \neq 0$ has an outer derivation (see Proposition 6.5 below).

Obviously, a finite-dimensional Leibniz algebra $L$ is semisimple if its maximal solvable ideal is equal to Leib $(L)$. Semisimple Leibniz algebras have studied in $[2,33,45,83]$ and others. In this way we prove in Section 8 the next result.

Theorem 5.2. If $L$ is a $D$-prime (respectively $D$-semisimple or $D$-simple) Leibniz algebra, then $D / A D e r L$ is prime (respectively semisimple or simple) Lie algebra, where $\mathrm{ADer} L:=\{\delta \in \operatorname{Der} L \mid \delta(L) \subseteq \operatorname{rann} L\}$.

A linear mapping $T: L \rightarrow L$ is called a multiplier of a Leibniz algebra $L$ whenever

$$
T(x y)=T(x) y
$$

for all $x, y \in L$. The set of all multipliers of $L$ will be denoted by $\mathrm{M} L$. Obviously that, for any $T \in \mathrm{M} L,(T, 0) \in \operatorname{IGDer} L$ and so

$$
\mathrm{M} L \subseteq \mathrm{IGDer} L \subseteq \operatorname{GDer} L
$$

Moreover, $\mathrm{M} L$ is an ideal of the Lie ring GDer $L$. In Section 8 we will prove the following

Theorem 5.3. Let $L$ be a Leibniz algebra. Then the following hold:

(1) if $\operatorname{Der} L / \operatorname{ADer} L$ is a semisimple (respectively prime, simple or primary) Lie algebra, then $L / \operatorname{rann} L$ is a $\bar{G}$-semiprime (respectively $\bar{G}$-prime, $\bar{G}$ simple or $\bar{G}$-primary) Lie algebra,

(2) if $\operatorname{Inn} L / \operatorname{AInn} L$, where $\operatorname{AInn} L=\operatorname{ADer} L \cap \operatorname{Inn} L$, is a semisimple (respectively prime, simple or primary) Lie algebra, then $L / \operatorname{rann} L$ is a semisimple (respectively prime, simple or primary) Lie algebra.

For basic definitions and properties of Leibniz and Lie algebras we refer to $[2,7,48,49,59]$. 


\section{Properties of Derivation Algebras}

At first we will present some information about derivation algebras. If $A \subseteq L$, then

$$
\operatorname{Inn}_{A} L:=\left\{r_{a} \mid a \in A\right\}
$$

and, in particular, $\operatorname{Inn} L=\operatorname{Inn}_{L} L$.

Lemma 6.1. Let $L$ be a Leibniz algebra, $A$ its ideal. Then the following hold:

(i) if $A$ is a $\Delta$-ideal of $L$, then $\operatorname{Inn}_{A} L$ is an ideal of $D$,

(ii) $\operatorname{Inn}_{A} L$ is an ideal of $\operatorname{Inn} L$,

(iii) $\operatorname{Inn}_{A} L=0$ if and only if $A \subseteq \operatorname{rann} L$,

(iv) $\operatorname{Inn} L=0$ if and only if $L^{2}=0$,

(v) $\operatorname{Inn}_{A} L=\operatorname{Inn} L$ if and only if $L=A+\operatorname{rann} L$,

(vi) $\operatorname{rann} L$ is a $D$-ideal of $L$,

(vii) there is the Lie algebra isomorphism

$$
\operatorname{Inn} L \ni r_{a} \mapsto a+\operatorname{rann} L \in L / \operatorname{rann} L,
$$

(viii) if $\Phi$ is an ideal of $\operatorname{Inn} L$, then

$$
\Delta_{\Phi}=\left\{a \in L \mid r_{a} \in \Phi\right\}
$$

is an ideal of $L$,

(ix) if $\Phi$ is an ideal of $D$, then $\Delta_{\Phi}$ is a D-ideal of $L$,

(x) if $B, C \subseteq L$, then $\left[\operatorname{Inn}_{B} L, \operatorname{Inn}_{C} L\right]=\operatorname{Inn}_{C B} L$.

Proof. By routine calculations.

Lemma 6.2. Let $A$ be a Leibniz algebra and $\Phi$ an ideal of $D$. Then we have:

(i) $\mathrm{ADer} L$ is an ideal of $D$,

(ii) $[\Phi, \operatorname{Inn} L]=0$ if and only if $\Phi \subseteq \operatorname{ADer} L$,

(iii) if $\Phi \cap \operatorname{Inn} L=0$, then $\Phi \subseteq \operatorname{ADer} L$.

Proof. (i) Immediately.

(ii) Let $\delta \in \Phi$ and $a \in L$.

Sufficiency. Since

$$
r_{\delta(a)}=\left[\delta, r_{a}\right]
$$

and $\left[\delta, r_{a}\right]=0$, we deduce that $\delta(a) \in \operatorname{rann} L$.

Necessity. Inasmuch $r_{\delta(a)}=0$, we conclude that the assertion holds in view of Eq. (6.1)).

(iii) It follows from (ii). 
Corollary 6.3. Let $L$ be a Leibniz algebra. Then $\operatorname{Inn} L$ is a simple (respectively prime, semisimple or primary) Lie algebra if and only if $L$ is a simple (respectively prime, semisimple or primary) Leibniz algebra.

Proof of Proposition 5.1. (1) $\Rightarrow(2)$. Let $D$ be a simple Lie algebra. If $\operatorname{Inn} L=0$, then $L^{2}=0$ and any endomorphism of the additive group $L^{+}$is a derivation of $L$. If $p$ is a prime, then

$$
\begin{aligned}
E_{p}=\left\{\sigma_{m}: L^{+} \rightarrow L^{+} \mid \sigma_{m}(a)=m a, \text { where } a \in L\right. \\
\quad \text { and } p \text { is a divisor of an integer } m\},
\end{aligned}
$$

is an ideal of $D$. Therefore $E_{p}=D$ if the characteristic charakt $\mathbb{K}=0$ or $E_{q}=D=E_{r}$ if the characteristic charakt $\mathbb{K}=p$, where $p, q, r$ are pair wise distinct primes. This leads to a contradiction. Hence

$$
0 \neq \operatorname{Inn} L=D \text {. }
$$

Then $L / \operatorname{rann} L$ is a simple Lie algebra by Corollary $6.3, L=L^{2}+\operatorname{rann} L$ and

$$
D=[D, D]=[\operatorname{Inn} L, \operatorname{Inn} L]=\operatorname{Inn} L .
$$

$(2) \Rightarrow(1)$. By Corollary 6.3, $\operatorname{Inn} L$ is a simple Lie algebra. Consider three cases.

(2a) Let $D$ be a prime Lie algebra and $A, B$ be $D$-ideals of $L$ such that

$$
A B \subseteq \operatorname{rann} L .
$$

Then

$$
\left[\operatorname{Inn}_{B} L, \operatorname{Inn}_{A} L\right]=0
$$

by Lemma $6.1(x)$ and, as a consequence, $\operatorname{Inn}_{B} L=0$ or $\operatorname{Inn}_{A} L=0$. This means that $B \subseteq \operatorname{rann} L$ or $A \subseteq \operatorname{rann} L$. Thus $L$ is a $D$-prime Leibniz algebra.

(2b) If $D$ is a semisimple Lie algebra, then we can obtain the assertion by the same argument as in the part $(2 a)$.

(2c) Assume that $D$ is a primary Lie algebra and $A, B$ are $D$-ideals of $L$

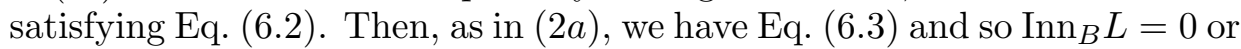
$\operatorname{Inn}_{A} L$ is a nilpotent ideal of $D$. Consequently $B \subseteq \operatorname{rann} L$ or $A^{n} \subseteq \operatorname{rann} L$. Hence $L$ is a $D$-primary Leibniz algebra.

Lemma 6.4. Let $L$ be a Leibniz algebra and $M$ be its ideal of codimension 1. If $0 \neq Z(L) \subseteq M$, then $Z(M)$ is an ideal of $L$ and $Z(M) \neq L \cdot Z(M)$.

Proof. It is easy to see that $L=M \oplus a \mathbb{K}$ is a direct sum of subspaces, where $a \in L$. Since

$$
m(u x)=0, \quad m(x u)=0, \quad(u x) m=0, \quad(x u) m=0
$$


for all $x \in L, m \in M$ and $u \in Z(M)$, we deduce that $Z(M)$ is an ideal of $L$. Moreover,

$$
l_{a}: Z(M) \ni u \mapsto a u \in Z(M)
$$

is an endomorphism of the additive group $Z(M)^{+}$.

Since $0 \neq Z(L) \subseteq Z(M)$, we see that the kernel $\operatorname{Kerl}_{a} \neq 0$ and so

$$
\operatorname{dim} Z(M)>\operatorname{dim}(a \cdot Z(M)) .
$$

Hence $L \cdot Z(M) \neq Z(M)$.

Proposition 6.5. Let $L$ be a finite-dimensional Leibniz algebra such that $Z(L) \neq 0$ and $L^{2} \neq L$. Then $L$ has an outer derivation.

Proof. Since $L^{2} \neq L$, we deduce that there exists a subspace $M$ of codimension 1 of $L$ such that $L^{2} \subseteq M$ and $L=M \oplus a \mathbb{K}$ is a direct sum of subspaces for some $a \in L$. Obviously that $M$ is an ideal of $L$. Suppose that $0 \neq z_{0} \in L$ and there exists a linear map

$$
\delta: L \ni m+\lambda a \mapsto \lambda z_{0} \in L,
$$

where $\lambda \in \mathbb{K}, \delta(a)=z_{0}$ and $\delta(m)=0$ for any $m \in M$. If, moreover, $\delta=r_{u}$ is an inner derivation for some $u \in L$, then $0=\delta(M)=M u$ and

$$
z_{0}=\delta(a)=r_{u}(a)=a u .
$$

1) If $Z(L) \nsubseteq M, z_{0} \in Z(L) \backslash M$ and $a \in Z(L)$, then $0 \neq \delta \in \operatorname{Der} L$ and $z_{0}=a u \in M$ by Eq. (6.4), a contradiction.

2) If $Z(L) \nsubseteq L^{2}$ and $z_{0} \in Z(L) \backslash L^{2}$, then $0 \neq \delta \in \operatorname{Der} L$ and $z_{0}=a u \in L^{2}$, a contradiction.

3) Assume that $Z(L) \subseteq L^{2}$ and so $Z(L) \subseteq Z(M)$.

a) Suppose $Z(M) \neq Z(L)$ and $z_{0} \in Z(M) \backslash Z(L)$. Then $0 \neq \delta \in \operatorname{Der} L$. If $u=m_{0}+\lambda_{0} a$ for some $m_{0} \in M$ and $\lambda_{0} \in \mathbb{K}$, then

$$
z_{0} u=\lambda_{0} z_{0} a, \quad u z_{0}=\lambda_{0} a z_{0}
$$

and

$$
z_{0} u+u z_{0}=\lambda_{0} \delta\left(a^{2}\right)=0 .
$$

This yields that $u z_{0}=-z_{0} u=0$ and consequently $z_{0} \in Z(L)$, which gives a contradiction.

Now assume that $Z(L)=Z(M)$.

b) If $z_{0} \in Z(M) \backslash L \cdot \operatorname{rann} M$, then $0 \neq \delta \in \operatorname{Der} L$. Indeed, $a^{2} \in M$ and therefore $\delta\left(a^{2}\right)=0$. Then

$$
\begin{aligned}
\delta\left(\left(m_{1}+\lambda_{1} a\right)\right. & \left.\left(m_{2}+\lambda_{2} a\right)\right)=0=\lambda_{1} \lambda_{2} \delta\left(a^{2}\right) \\
& =\lambda_{1} \lambda_{2} \delta(a) a+\lambda_{1} \lambda_{2} a \delta(a)= \\
& =\lambda_{1} z_{0}\left(m_{2}+\lambda_{2} a\right)+\left(m_{1}+\lambda_{1} a\right) \lambda_{2} z_{0}= \\
& =\delta\left(m_{1}+\lambda_{1} a\right) \cdot\left(m_{2}+\lambda_{2} a\right)+\left(m_{1}+\lambda_{1} a\right) \cdot \delta\left(m_{2}+\lambda_{2} a\right)
\end{aligned}
$$


for any $m_{1}, m_{2} \in M$ and $\lambda_{1}, \lambda_{2} \in \mathbb{K}$. Moreover, $z_{0}=a u \in L \cdot \operatorname{rann} M$, which gives a contradiction.

c) Now assume that $Z(M)=L \cdot \operatorname{rann} M$. In view of Lemma 6.4 ,

$$
Z(M) \neq L \cdot Z(M)
$$

and so $Z(M) \neq \operatorname{rann} M$. If $z_{0} \in Z(M) \backslash \operatorname{rann} M$, then $0 \neq \delta \in \operatorname{Der} L$ and $z_{0}=a u \in \operatorname{rann} M$, which gives a contradiction.

It now follows from 1)-3) that $\delta$ is an outer derivation of $L$.

Corollary 6.6. If $L$ is a finite-dimensional nilpotent Leibniz algebra, then it admits an outer derivation.

\section{Generalized Derivations}

Lemma 7.1. Let $L$ be a Leibniz algebra. Then:

(i) GDerL is a Lie ring with respect to the point-wise addition "+ " and the point-wise Lie multiplication "[-, -]" given by the rules

$$
(H+K)(x)=H(x)+K(x)
$$

and

$$
[H, K](x)=H(K(x))-K(H(x))
$$

for all $x \in L$ and $H, K \in \mathrm{GDer} L$;

(ii) if $A$ is a D-ideal of $L$, then

$\mathrm{I}_{\mathrm{A}} \operatorname{GDer} L=\left\{F \in \operatorname{GDer} L \mid F\right.$ is associated with some $\left.r_{a} \in \operatorname{Inn}_{A} L\right\}$

is an ideal of GDerL. In particular,

$$
\text { IGDer } L=\mathrm{I}_{\mathrm{L}} \text { GDer } L, \quad \mathrm{M} L=\mathrm{I}_{\mathrm{O}} \text { GDer } L .
$$

(iii) GDer $L=\mathrm{M} L+\operatorname{Der} L$, where $\mathrm{M} L$ is an ideal of GDer $L$, and

$$
\mathrm{M} L \bigcap \operatorname{Der} L \subseteq \operatorname{ADer} L ;
$$

(iv) IGDer $L=\mathrm{M} L+\operatorname{Inn} L$, where $\mathrm{M} L$ is an ideal of $\mathrm{IGDer} L$, and

$$
\mathrm{M} L \bigcap \operatorname{Inn} L \subseteq \operatorname{ADer} L ;
$$

(v) if $(F, \delta),(F, d) \in \operatorname{GDer} L$, then $\delta+\operatorname{ADer} L=d+\operatorname{ADer} L$.

Proof. Assume that $(F, \delta),(K, d) \in \operatorname{GDer} L, T \in \mathrm{M} L$ and $x, y \in L$.

(i) We see that $(F-K, \delta-d) \in \operatorname{GDer} L$,

$$
\begin{aligned}
{[F, K](x y) } & =F(K(x) y+x d(y))-K(F(x) y+x \delta(y))= \\
& =[F, K](x) y+x[\delta, d](y)
\end{aligned}
$$

and so $([F, K],[\delta, d]) \in \operatorname{GDer} L$.

(ii) Evident. 
(iii) The equality

$$
[F, T](x y)=[F, T](x) y
$$

implies that $[F, T] \in \mathrm{M} L$ and, as a consequence, $\mathrm{M} L$ is an ideal of GDer $L$. From

$$
(\delta-F)(x y)=\delta(x) y+x \delta(y)-F(x) y-x \delta(y)=(\delta-F)(x) y
$$

it follows that $\delta-F \in \mathrm{M} L$. If $g \in \operatorname{Der} L \cap \mathrm{M} L$, then

$$
g(x) y=g(x y)=g(x) y+x g(y) .
$$

Hence $x g(y)=0$ whih implies that $g(L) \subseteq \operatorname{rann} L$.

(iv) By the same argument as in the part (iii).

(v) If $(F, \delta),(F, d) \in \operatorname{GDer} L$ for some $\delta, d \in \operatorname{Der} L$, then

$$
x \delta(y)=x d(y)
$$

and consequently $x(\delta-d)(y)=0$. This means that $(\delta-d)(L) \subseteq \operatorname{rann} L$.

Lie algebras $L$ with abelian derivation algebras $\operatorname{Der} L$ was studied by S. Tôgô [92].

Lemma 7.2. Let $L$ be a Leibniz algebra and $(F, d) \in \operatorname{GDer} L$. Then we have:

(i) if $F=0$, then $d \in \operatorname{ADer} L$,

(ii) if $d \in \mathrm{ADer} L$, then $F \in \mathrm{M} L$,

(iii) if GDer $L$ is an abelian Lie algebra, then $\operatorname{Der} L$ is abelian,

(iv) if $L \neq 0$, then $\operatorname{IGDer} L \neq 0$.

Proof. Assume that $x, y \in L$.

(i) In fact,

$$
0=F(x y)=F(x) y+x d(y)=x d(y)
$$

and so $d(y) \in \operatorname{rann} L$.

(ii) Since $F(x y)=F(x) y$, we deduce that $F \in \mathrm{M} L$.

(iii) We have $\operatorname{Der} L \subseteq$ GDer $L$ and therefore the assertion holds.

(iv) Straightforward.

Lemma 7.3. Let $L$ be a Leibniz algebra and $\left(H, r_{a}\right) \in \operatorname{IGDer} L$. Then the following hold:

(i) if $H=0$, then $a \in \operatorname{rann} L$,

(ii) if $a \in \operatorname{rann} L$, then $H \in \mathrm{M} L$,

(iii) if IGDer $L$ is an abelian Lie algebra, then $L^{2} \subseteq \operatorname{rann} L$,

(iv) if $L$ is abelian, then $\operatorname{IGDer} L=\mathrm{M} L$.

Proof. It is easy to check by using the same argument as in the proof of Lemma 7.2. 
Let $\Phi \subseteq \operatorname{GDer} L, \Gamma \subseteq \operatorname{Der} L$

$T_{\Phi}=\{d \in \operatorname{Der} L \mid$ there is $H \in \Phi$ that is associated with $d \in \operatorname{Der} L\}$,

$U_{\Gamma}=\{H \in \operatorname{GDer} L \mid H$ is associated with some $d \in \Gamma\}$,

$\Sigma_{\Phi}=\left\{a \in L \mid\right.$ there is $H \in \Phi$ that is associated with $\left.r_{a} \in \operatorname{Inn} L\right\}$.

Lemma 7.4. Let $L$ be a Leibniz algebra. If $\Phi$ is an ideal of GDer $L$ such that $[\Phi, \operatorname{IGDer} L]=0$, then $T_{\Phi} \subseteq \operatorname{ADer} L$ (and so $\Phi \subseteq \mathrm{M} L$ ).

Proof. Indeed, if $(F, d) \in \Phi$, then $r_{d(a)}=\left[d, r_{a}\right] \in \operatorname{ADer} L$ for any $a \in L$ and so $d(a) \in \operatorname{rann} L$.

Lemma 7.5. Let $L$ be a Leibniz algebra. Then the following hold:

(1) if $\Phi$ is an ideal of IGDer $L$ (respectively GDer $L$ ), then $\Sigma_{\Phi}$ is an ideal (respectively a D-ideal) of $L$,

(2) if $\Gamma$ is an ideal of $\operatorname{Der} L$, then $U_{\Gamma}$ is an ideal of $\operatorname{GDer} L$ (in particular, $\left.U_{0}=\mathrm{I}_{\mathrm{O}} \mathrm{GDer} L=\mathrm{M} L\right)$,

(3) if $\Phi$ is an ideal of GDer $L$, then $T_{\Phi}$ is an ideal of $\operatorname{Der} L$.

Proof. (1) Let $a, b \in \Sigma_{\Phi}, t \in L,\left(H, r_{a}\right),\left(K, r_{b}\right) \in \Phi,\left(S, r_{t}\right) \in \operatorname{IGDer} L$ and $(M, \delta) \in \mathrm{GDer} L$. Since

$$
\left(H-K, r_{a-b}\right),\left([M, H], r_{\delta(a)}\right),\left([H, S], r_{t a}\right),\left([S, H], r_{a t}\right) \in \Phi,
$$

we conclude that $a-b, \delta(a), t a, a t \in \Sigma_{\Phi}$ and therefore $\Sigma_{\Phi}$ is an ideal of $L$.

(2)-(3) By the same argument as in the part (1).

Lemma 7.6. Let $L$ be a Leibniz algebra and $A$ its ideal. Then the following conditions are equivalent:

(1) $\mathrm{I}_{\mathrm{A}} \mathrm{GDer} L \subseteq \mathrm{M} L$,

(2) $A \subseteq \operatorname{rann} L$,

(3) $\operatorname{Inn}_{A} L=0$.

Proof. For proof see Lemmas 6.1 and 7.1 .

Lemma 7.7. Let $L$ be a Leibniz algebra and $a \in L$. If $L$ is $D$-semisimple, then $r_{a} \in \mathrm{ADer} L$ if and only if $a \in \operatorname{rann} L$.

Proof. We have that

$$
L a=r_{a}(L) \subseteq \operatorname{rann} L .
$$

Moreover, $L \delta^{n}(a) \subseteq \operatorname{rann} L$ for any $\delta \in D$ and a non-negative integer $n$. If $l, t \in L$, then

$$
0=t\left(l \delta^{n}(a)\right)=(t l) \delta^{n}(a)-\left(t \delta^{n}(a)\right) l
$$


and so $\left(t \delta^{n}(a)\right) l \in L \delta^{n}(a)$. Hence

$$
L_{0}:=L a+\sum_{n=1}^{\infty} L \delta^{n}(a)
$$

is a $D$-ideal of $L$ and $L_{0} \cdot L_{0}=0$. Consequently $L a=0$.

Lemma 7.8. Let $L$ be a Leibniz algebra and $A$ its ideal. Then there exist Lie algebra isomorphisms:

(1) $\operatorname{Der} L / \operatorname{ADer} L \ni d+\operatorname{ADer} L \longmapsto F+\mathrm{M} L \in \operatorname{GDer} L / \mathrm{M} L$, where $(F, d) \in \operatorname{GDer} L$

(2) $\operatorname{Inn} L / \mathrm{AInn} L \ni r_{a}+\mathrm{A} \operatorname{Inn} L \longmapsto H+\mathrm{M} L \in \operatorname{IGDer} L / \mathrm{M} L$, where $\left(H, r_{a}\right) \in \mathrm{IGDer} L$.

Proof. Straightforward.

\section{ProOFS}

Proof of Theorem 5.2. (a) Let $L$ be a $D$-prime Leibniz algebra and $\Psi, \Omega$ be ideals of $D$ such that $[\Psi, \Omega]=0$. Then $\operatorname{rann} L=\operatorname{Leib}(L)$. If

$$
\Phi:=\Psi \bigcap \operatorname{Inn} L \quad \text { and } \quad \Lambda:=\Omega \bigcap \operatorname{Inn} L
$$

then

$$
\Phi=\operatorname{Inn}_{\Delta_{\Phi}} L, \quad \Lambda=\operatorname{Inn}_{\Delta_{\Lambda}} L, \quad[\Phi, \Lambda]=0 .
$$

Lemma $6.1(\mathrm{x})$ and (iii) imply that

$$
\Delta_{\Lambda} \Delta_{\Phi} \subseteq \operatorname{rann} L
$$

Since $\Delta_{\Lambda}$ and $\Delta_{\Phi}$ are $D$-ideals by Lemma $6.1(\mathrm{ix})$, we deduce that

$$
\Delta_{\Lambda} \subseteq \operatorname{rann} L \quad \text { or } \quad \Delta_{\Phi} \subseteq \operatorname{rann} L
$$

by the $D$-primeness of $L$. This gives that $\Lambda=0$ or $\Phi=0$ by Lemma 6.1 (iii). As a consequence of Lemma 6.2(iii), the quotient Lie algebra $D / \operatorname{ADer} L$ is prime.

(b) If $L$ is a $D$-semisimple Leibniz algebra, then we can obtain that $D / \operatorname{ADer} L$ is semisimple analogously as in (a).

(c) Assume that $L$ is a $D$-simple Leibniz algebra and $\Psi$ is an ideal of $D$. Then $\operatorname{rann} L=\operatorname{Leib}(L)$. If $\Phi:=\Psi \cap \operatorname{Inn} L$, then $\Delta_{\Phi}$ is a $D$-ideal of $L$ and so $\Delta_{\Phi} \subseteq \operatorname{rann} L$. By Lemma $6.1(\mathrm{iii}), \Phi=\operatorname{Inn}_{\Delta_{\Phi}} L=0$ and $\Psi \subseteq \operatorname{ADer} L$ by Lemma 6.1(iii). Thus $D / \operatorname{ADer} L$ is a simple Lie algebra.

Proposition 8.1. Let $L$ be a Leibniz algebra. Then the following hold: 
(1) if GDer $L / M L$ is a semisimple (respectively prime, simple or primary) Lie algebra, then $L / \operatorname{rann} L$ is a $\bar{G}$-semiprime (respectively $\bar{G}$-prime, $\bar{G}$-simple or $\bar{G}$-primary) Lie algebra;

(2) if IGDer $L / \mathrm{M} L$ is a semisimple (respectively prime, simple or primary) Lie algebra, then $L / \operatorname{rann} L$ is a semiprime (respectively prime, simple or primary) Lie algebra.

Proof. (1a) Assume that GDer $L / \mathrm{M} L$ is a prime Lie algebra and $A, B$ are $G$-ideals of $L$ such that $A B \subseteq \operatorname{rann} L$. Then

$$
\left[\mathrm{I}_{\mathrm{B}} \mathrm{GDer} L, \mathrm{I}_{\mathrm{A}} \mathrm{GDer} L\right] \subseteq \mathrm{M} L
$$

and so $\mathrm{I}_{\mathrm{B}} \mathrm{GDer} L \subseteq \mathrm{M} L$ or $\mathrm{I}_{\mathrm{A}}$ GDer $L \subseteq \mathrm{M} L$ what forces that $B \subseteq \operatorname{rann} L$ or $A \subseteq \operatorname{rann} L$ by Lemma 7.6. Hence $L / \operatorname{rann} L$ is a $\bar{G}$-prime Lie algebra.

(1b) If GDer $L / \mathrm{M} L$ is a semisimple Lie algebra, then we can prove by the same argument as in the case (1a).

(1c) Assume that GDer $L / \mathrm{M} L$ is a simple Lie algebra and $A$ is a $G$-ideal of $L$. By Lemma 7.1(ii), $\mathrm{I}_{\mathrm{A}}$ GDer $L$ is an ideal of GDer $L$ and so

$$
\operatorname{GDer} L=\mathrm{I}_{\mathrm{A}} \operatorname{GDer} L
$$

or

$$
\mathrm{M} L=\mathrm{I}_{\mathrm{A}} \mathrm{GDer} L
$$

what implies that $L=A+\operatorname{rann} L$ or $A \subseteq \operatorname{rann} L$. Consequently $L / \operatorname{rann} L$ is a $\bar{G}$-simple Lie algebra.

(1d) Let GDer $L / \mathrm{M} L$ be a primary Lie algebra and $A, B$ be $G$-ideals of $L$ satisfying the condition $A B \subseteq \operatorname{rann} L$.

Then (8.1) holds and so $\mathrm{I}_{\mathrm{A}} \mathrm{GDer} L \subseteq \mathrm{M} L$ or

$$
[\underbrace{\mathrm{I}_{\mathrm{B}} \mathrm{GDer} L, \ldots, \mathrm{I}_{\mathrm{B}} \mathrm{GDer} L}_{m \text { times }}] \subseteq \mathrm{M} L
$$

for some positive integer $m$. Therefore $A \subseteq \operatorname{rann} L$ or $B^{m} \subseteq \operatorname{rann} L$. Hence $L / \operatorname{rann} L$ is a $\bar{G}$-primary Lie algebra.

(2) By the analogues argument as in the proof of the part (1).

Proof of Theorem 5.3. This theorem is a consequence of Proposition 8.1 and Lemma 7.8.

9. Supplement: The Classical Poisson manifolds approach REVISITED

\subsection{Poisson structures on noncommutative functional manifolds.}

It is interesting to look at the construction of the Hamiltonian operators presented above and revisit it from the standard point of view, considering them as those defined on the naturally associated $[1,4,22,20,68,70,78]$ cotangent space $T^{*}(M)$ to some linear functional manifold $M \simeq \widetilde{\mathbb{A}}^{*} \simeq \widetilde{\mathbb{A}}$. 
Then, a Hamiltonian operator on $M$ is defined [1] as a smooth mapping $\vartheta: M \rightarrow \operatorname{Hom}\left(T^{*}(M) ; T(M)\right)$, such that for any fixed $u \in M$ the bracket

$$
\{f, g\}:=(\nabla f(u), \vartheta(u) \nabla g(u)),
$$

where $f, g: M \rightarrow \mathbb{K}$ are arbitrary smooth mappings from the functional space $\mathcal{D}(M) \simeq \mathcal{F}_{\widetilde{A}}(u)$, satisfies the Jacobi identity. The bracket (9.1) is determined on $M$ by means of the natural convolution $(\cdot, \cdot)$ on the product $T^{*}(M) \times T(M)$, and respectively, the gradient $\nabla f(u) \in T^{*}(M)$ of a function $f \in \mathcal{D}(M)$ is calculated as

$$
(\nabla f(u), h):=d f[u+\varepsilon h] /\left.d \varepsilon\right|_{\varepsilon=0}
$$

for any $h \in T(M)$. It is well known $[43,58]$ that a linear operator

$$
\vartheta(u): T^{*}(M) \rightarrow T(M),
$$

determined at any point $u \in M$, is Hamiltonian iff the suitably defined [43] Schouten-Nijenhuis bracket

$$
[[\vartheta(u), \vartheta(u)]]=0
$$

identically on $M$. Namely, this condition (9.3) was used in the investigations $[43,90]$ to formulate criteria for the operator $\vartheta(u): T^{*}(M) \rightarrow T(M)$ to be Hamiltonian on the functional manifold $M$. Yet these criteria appear to be very complicated and involve a large amount of cumbersome calculations even in the case of fairly simple differential expressions. So, we have reanalyzed this problem from a slightly different point of view. First, recall that the Jacobi identity for the bracket (9.1) is completely equivalent to the fact that the bracket operator defined as $D_{f}(g):=\{f, g\}$ for a fixed $f \in \mathcal{D}(M)$ and arbitrary $g \in \mathcal{D}(M)$ acts as a derivation on the space $(\mathcal{D}(M) ;\{\cdot, \cdot\})$ :

$$
D_{f}\{g, h\}=\left\{D_{f}(g), h\right\}+\left\{g, D_{f}(h)\right\},
$$

where $g, h \in \mathcal{D}(M)$ are taken arbitrary. This can be easily reformulated as follows: take any element $\varphi \in T^{*}(M)$, such that the Fréchet derivative $\varphi^{\prime}(u)=\varphi^{\prime, *}(u)$ at any $u \in M$ with respect to the convolution $(\cdot, \cdot)$ on $T^{*}(M) \times T(M)$, and construct a vector field $K: M \rightarrow T(M)$ as

$$
K(u):=\vartheta(u) \varphi(u)
$$

Then the derivation condition (9.4) can be equivalently rewritten $[1,68$, $20,70,78]$ as the strong Lie derivative

$$
L_{K} \vartheta:=\vartheta^{\prime} \cdot K-\vartheta K^{\prime, *}-K^{\prime} \vartheta=0
$$

along the vector field $K(u)=\vartheta(u) \varphi(u) \in T(M)$ at any $u \in M$ for all "selfadjoint" elements $\varphi \in T^{*}(M)$. Equivalently, a given linear skew-symmetric operator $\vartheta(u): T^{*}(M) \rightarrow T(M), u \in M$, is Hamiltonian iff the Lie derivative (9.5) vanishes for all "self-adjoint" elements $\varphi \in T^{*}(M)$. Moreover, 
as was observed in [64], it suffices to check the condition (9.5) only on the subspace of elements $\varphi \in T^{*}(M)$ satisfying the condition $\varphi^{\prime}(u)=0$ for any $u \in M$.

As an example, one can check that a skew-symmetric matrix-differential operator on $M$ of the form

$$
\vartheta(u):=\sigma(u) D_{x}+D_{x} \sigma^{\top}(u),
$$

where, an $n$-dimensional square matrix

$$
\sigma(u):=\left(\sum_{s=1}^{n} u_{s} \sigma_{i j}^{s} \mid i, j=\overline{1, n}, n \in \mathbb{Z}_{+}, u \in M\right)
$$

satisfies the condition (9.5) iff the linearly independent elements from

$$
\operatorname{span}_{\mathbb{K}}\left\{e_{j} \in \mathbb{A} \mid j=\overline{1, n}\right\}
$$

generate the finite dimensional nonassociative Balinsky-Novikov algebra (4.2) and satisfy the conditions $e_{i} \circ e_{j}=\sum_{s=1}^{n} \sigma_{i j}^{s} e_{s}$ for all $i, j=\overline{1, n}$. Similarly, one can verify that the skew-symmetric inverse-differential operator

$$
\vartheta(u):=\sigma(u) D_{x}^{-1}+D_{x}^{-1} \sigma(u)^{\top},
$$

where, as above $\sigma(u):=\left(\sum_{s=1}^{n} u_{s} \sigma_{i j}^{s} \mid i, j=\overline{1, n}, n \in \mathbb{Z}_{+}, u \in M\right)$, the sign " $T$ " means the usual matrix transposition, is Hamiltonian iff the basic nonassociative algebra $\mathbb{A}:=\operatorname{span}\left\{e_{j}: j=\overline{1, n}\right\}$ coincides with the right Leibniz algebra (4.3) and the condition $e_{i} \circ e_{j}=\sum_{s=1}^{n} \sigma_{i j}^{s} e_{s}$ holds for any $i, j=\overline{1, n}$. The skew-symmetric inverse-differential operator (9.7) can be naturally generalized to the expression

$$
\vartheta(u):=D_{x} \sigma(u) D_{x}^{-1}-D_{x}^{-1} \sigma(u)^{\top} D_{x},
$$

which can be rewritten as

$$
\vartheta(u)=\sigma\left(D_{x} u\right) D_{x}^{-1}+D_{x}^{-1} \sigma\left(D_{x} u\right)^{\top}+\sigma(u)-\sigma(u)^{\top} .
$$

The condition (9.5) for the operator (9.8) to be Hamiltonian reduces to the constraints on the related nonassociative algebra

$$
\mathbb{A}:=\operatorname{span}_{\mathbb{K}}\left\{e_{j}: j=\overline{1, n}\right\}
$$

exactly coinciding with that of (4.4), and analyzed in some detail in Section 3 . 
As it was already mentioned, based on the matrix representations of the right Leibniz algebra (4.3) and the new nonassociative Riemann algebra (4.5), one can construct many nontrivial Hamiltonian operators

$$
\vartheta(u): \mathcal{L}_{\widetilde{\mathbb{A}}} \rightarrow \mathcal{L}_{\widetilde{\mathbb{A}}}
$$

on the associated weak Lie algebra $\mathcal{L}_{\widetilde{\mathbb{A}}}$, related with diverse types of nonassociative algebras $\mathbb{A}$. These Hamiltonian operators prove to be very useful [21, 80, 81] for describing a wide class of multicomponent hierarchies of integrable Riemann type hydrodynamic systems and their various physically reasonable reductions.

\subsection{Poisson structures on manifolds generated by associative non-} commutative algebras. Proceed now to a slightly generalized construction of Hamiltonian operators on a phase space, generated by associative noncommutative algebra $A$-valued matrices, which was first studied in $[25,35,76,78]$ in case of the noncommutative operator algebras and continued later in $[62,51,52,53,62,66,67,71]$ in case of general associative noncommutative algebras. This natural and simple generalization appeared to be very useful $[5,6,94,96,62,66,67]$ for describing a wide class of new Lax type integrable nonlinear Hamiltonian systems on associative noncommutative algebras, interesting for diverse applications in modern quantum physics.

We start here with a free associative noncommutative algebra

$$
A=\mathbb{K}\left\langle u_{1}, u_{2}, \ldots, u_{m}\right\rangle,
$$

generated by a finite set of elements $\left\{u_{j} \in A: j=\overline{1, m}\right\}$, and define its "abelianization" $A_{\natural}:=A /[A, A]$ and the projection $\pi: A \rightarrow A_{\natural}$, where $[A, A]:=\{u v-v u \in A: u, v \in A\}$. Consider now a naturally related with $A$ n-dimensional matrix Lie algebra $\mathcal{G}:=\operatorname{gl}(n ; A)$ over the field $\mathbb{K}$ with entries in $A$ subject to the usual matrix commutator $[a \mid b]:=a b-b a$ for all $a, b \in \mathcal{G}$. Being first interested in the Lie-algebraic studying [22, 20, 38, 85] of co-adjont orbits on the adjoint space $\mathcal{G}^{*}$, let us construct a bi-linear form $\langle\cdot \mid \cdot\rangle: \mathcal{G} \times \mathcal{G} \rightarrow A_{\natural}$ on the Lie algebra $\mathcal{G}$ by means of the trace-type expression

$$
\langle a \mid b\rangle:=\pi \operatorname{tr}\left(a^{\top} b\right)
$$

for any $a, b \in \mathcal{G}$. The following important lemma holds.

Lemma 9.3. The bilinear form (9.9) on $\mathcal{G}$ is symmetric, nondegenerate and ad-invariant. 
Proof. Symmetricity. We have:

$$
\begin{aligned}
\langle a \mid b\rangle & =\sum_{i, j=\overline{1, n}} \pi\left(a_{i j} b_{i j}\right) \\
& =\sum_{i, j=\overline{1, n}} \pi\left(a_{i j} b_{i j}-b_{i j} a_{i j}\right)+\sum_{i, j=\overline{1, n}} \pi\left(b_{i j} a_{i j}\right) \\
& =\sum_{i, j=\overline{1, n}} \pi\left(b_{i j} a_{i j}\right)=\langle b \mid a\rangle
\end{aligned}
$$

for any $a, b \in \mathcal{G}$.

Nondegeneracy. Assume that $\langle a \mid b\rangle=0_{\natural} \in A_{\natural}$ for a fixed $a \in \mathcal{G}$ and all $b \in \mathcal{G}$. To state that $\mathrm{a}=0$, let us put then $\mathrm{b}=\mathrm{a}$ and obtain

$$
\langle a \mid a\rangle=\sum_{i, j=1, n} \pi\left(a_{i j} a_{i j}\right)=0_{\natural} .
$$

Taking into account that the associative algebra is generated by the finite set of elements $\left\{u_{j} \in A \mid j=\overline{1, m}\right\}$, it is easy to deduce from $n^{2}$ expansions of elements

$$
\begin{aligned}
& a_{i j}:=c_{k(i . j)}=\sum_{\left|s^{(1)}\right| \in \mathbb{Z}_{+}} \sum_{\sigma_{1} \in \sigma_{n}} C_{\left(k ; \sigma_{1}(1), \sigma_{1}(2), \ldots, \sigma_{1}(m)\right)}^{\left(s_{1}^{(1)} s_{2}^{(1)} \ldots s_{m}^{(1)}\right)}\left(u_{\sigma_{1}(1)}^{s_{1}^{(1)}} u_{\sigma_{1}(2)}^{s_{2}^{(1)}} \ldots u_{\sigma_{1}(m)}^{s_{m}^{(1)}}\right)+
\end{aligned}
$$

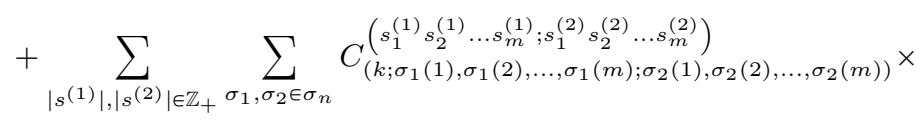

$$
\begin{aligned}
& \times\left(u_{\sigma_{1}(1)}^{s_{1}^{(1)}} u_{\sigma_{1}(2)}^{s_{2}^{(1)}} \ldots u_{\sigma_{2}(m)}^{s_{m}^{(1)}}\right) \times\left(u_{\sigma_{1}(1)}^{s_{1}^{s_{2}}} u_{\sigma_{2}(2)}^{s_{2}^{(2)}} \ldots u_{\sigma_{2}(m)}^{s_{m}^{(2)}}\right)+\ldots
\end{aligned}
$$

from $A$ that the sum

$$
\sum_{k=\overline{1, n}} \pi\left(c_{k} c_{k}\right)=0_{\natural}
$$

iff $c_{k}=0$ for all $k=\overline{1, n^{2}}$. Indeed, the sum of (9.11) under the $\pi$-mapping can be now rewritten, respectively, as

$$
\begin{aligned}
\sum_{k=1, n^{2}}\left(c_{k} c_{k}\right)= & \sum_{\left|s^{(1)}\right| \in \mathbb{Z}_{+}} \sum_{\sigma_{1} \in \sigma_{n}} D_{\left(\sigma_{1}(1), \sigma_{1}(2), \ldots, \sigma_{1}(m)\right)}^{\left(s_{1}^{(1)} s_{1}^{(1)} \ldots s_{1}^{(1)}\right)}\left(u_{\sigma_{1}(1)}^{s_{1}^{(1)}} u_{\sigma_{1}(2)}^{s_{2}^{(1)}} \ldots u_{\sigma_{1}(m)}^{s_{m}^{(1)}}\right)+ \\
+ & \sum_{\left|s^{(1)}\right|,\left|s^{(2)}\right| \in \mathbb{Z}_{+}} \sum_{\sigma_{1}, \sigma_{2} \in \sigma_{n}} D_{\left(\sigma_{1}(1), \sigma_{1}(2), \ldots, \sigma_{1}(m) ; \sigma_{2}(1), \sigma_{2}(2), \ldots, \sigma_{2}(m)\right)}^{\left(s_{1}^{(1)} s_{2}^{(1)} \ldots s_{m}^{(1)} ; s_{1}^{(2)} s_{2}^{(2)} \ldots s_{m}^{(2)}\right)} \times \\
& \times\left(u_{\sigma_{1}(1)}^{s_{1}^{(1)}} u_{\sigma_{1}(2)}^{s_{2}^{(1)}} \ldots u_{\sigma_{2}(m)}^{s_{m}^{(1)}}\right) \times\left(u_{\sigma_{1}(1)}^{s_{2}^{(2)}} u_{\sigma_{2}(2)}^{s_{2}^{(2)}} \ldots u_{\sigma_{2}(m)}^{s_{m}^{(2)}}\right)+\ldots
\end{aligned}
$$

with some $D$-coefficients from $\mathbb{K}$ for all $\sigma_{j} \in S_{n}$, depending quadratically on coefficients of expansions, staying at uniform and symmetric basis elements 
of the algebra $A$. As the $\pi$-mapping sends all of them, by definition, to zero, the resulting system (9.11) reduces to the set of algebraic equations

$$
\begin{gathered}
D_{\left(\sigma_{1}(1), \sigma_{1}(2), \ldots, \sigma_{1}(m)\right)}^{\left(s_{1}^{(1)} s_{2}^{(1)} \ldots s_{m}^{(1)}\right)}=0, \\
D_{\left(\sigma_{1}(1), \sigma_{1}(2), \ldots, \sigma_{1}(m) ; \sigma_{2}(1), \sigma_{2}(2), \ldots, \sigma_{2}(m)\right)}^{\left(s_{1}^{(1)} s_{2}^{(1)} \ldots s_{m}^{(1)} ; s_{1}^{(2)} s_{2}^{(2)} \ldots s_{m}^{(2)}\right)}=0,
\end{gathered}
$$

reducing successively for all $\sigma_{j} \in S_{n}$ to the conditions

$$
\begin{gathered}
C_{\left(k ; \sigma_{1}(1), \sigma_{1}(2), \ldots, \sigma_{1}(m)\right)}^{\left(s_{1}^{(1)} s_{2}^{(1)} \ldots s_{m}^{(1)}\right)}=0, \\
C_{\left(k ; \sigma_{1}(1), \sigma_{1}(2), \ldots, \sigma_{1}(m) ; \sigma_{2}(1), \sigma_{2}(2), \ldots, \sigma_{2}(m)\right)}^{\left(s_{1}^{(1)} s_{2}^{(1)} \ldots s_{m}^{(1)} ; s_{1}^{(2)} s_{2}^{(2)} \ldots s_{m}^{(2)}\right)}=0,
\end{gathered}
$$

being equivalent to the equalities $c_{k}=0$ for all $k=\overline{1, n^{2}}$.

As a simple consequence from Lemma 9.3 one derives the following proposition.

Proposition 9.4. The constructed Lie algebra $\mathcal{G}$ is ad-invariant and $\pi$ metrized.

Proof. Really, from the symmetry property (9.10) one easily obtains that

$$
\langle\mathrm{a} \mid[\mathrm{b}, \mathrm{c}]\rangle=\langle[\mathrm{a}, \mathrm{b}] \mid \mathrm{c}\rangle
$$

modulo $\pi$-mapping for any elements $a, b$ and $\mathrm{c} \in \mathcal{G}$. As the bilinear form (9.9) is non-degenerate, one has $\mathcal{G}^{*} \simeq \mathcal{G}$, that jointly with the ad-invariance property (9.12) means that the Lie algebra $\mathcal{G}$ is metrized.

Being interested in constructing integrable noncommutative dynamical systems on the algebra $A$, we need to introduce into our analysis a "spectral" parameter $\lambda \in \mathbb{C}$, responsible for the existence of infinite hierarchies of the corresponding dynamical systems invariants, guaranteeing their integrability. This wil be done in next Section, devoted to the Lie-algebraic analysis on loop-Lie-algebras, related with the Lie algebra $\mathcal{G}$, introduced above.

Consider now the Lie algebra $\{\mathcal{G},[\cdot, \cdot]\}$, constructed above, and the related loop Lie algebra

$$
\left\{\tilde{\mathcal{G}}:=\mathcal{G} \otimes \mathbb{C}\left\{\left\{\lambda, \lambda^{-1}\right\}\right\},[\cdot, \cdot]\right\}
$$


of the corresponding $\mathcal{G}$-valued Laurent series with respect to the parameter $\lambda \in \mathbb{C}$,

$$
\tilde{\mathcal{G}}:=\cup_{N \in \mathbb{Z}}\left\{\tilde{a}=\sum_{j \leqslant N} a_{j} \lambda^{j} \mid a_{j} \in \mathcal{G}, j=\overline{1, N}\right\},
$$

and define on it the corresponding to (9.9) modulo $\pi$-mapping bilinear form $(\cdot \mid \cdot): \tilde{\mathcal{G}} \times \tilde{\mathcal{G}} \rightarrow A:$

$$
(\tilde{a} \mid \tilde{b}):=r e s_{\lambda}\langle\tilde{m} a \mid \tilde{b}\rangle
$$

for any elements $\tilde{a}, \tilde{b} \in \tilde{\mathcal{G}}$. It is easy to observe that the bilinear form (9.13) is also symmetric and non-degenerate. Thus, the following proposition holds.

Proposition 9.5. The loop Lie algebra $\tilde{\mathcal{G}}$ is ad-invariant and $\pi$-metrized.

As the loop Lie algebra $\tilde{\mathcal{G}}$ allows natural direct sum splitting $\tilde{\mathcal{G}}=\tilde{\mathcal{G}}_{+} \oplus \tilde{\mathcal{G}}$ into two Lie subalgebras $\tilde{\mathcal{G}}_{+}$and $\tilde{\mathcal{G}}_{-}$, where

$$
\begin{gathered}
\tilde{\mathcal{G}}_{+}: \bigcup_{N \in \mathbb{Z}_{+}}\left\{\tilde{a}=\sum_{j=\overline{0, N}} a_{j} \lambda^{j} \mid a_{j} \in \mathcal{G}, j=\overline{1, N}\right\}, \\
\tilde{\mathcal{G}}_{-}:=\bigcup_{N \in \mathbb{Z}_{+}}\left\{\tilde{a}=\sum_{j \in \mathbb{Z}_{+}} a_{j} \lambda^{-(j+1)} \mid a_{j} \in \mathcal{G}, j \in \mathbb{Z}_{+}\right\},
\end{gathered}
$$

their adjoint spaces with respect to the bilinear form (9.13)) split the adjoint loop space $\tilde{\mathcal{G}}^{*}=\tilde{\mathcal{G}}_{+}^{*} \oplus \tilde{\mathcal{G}}_{-}^{*}$ and satisfy the equivalences

$$
\tilde{\mathcal{G}}_{+}^{*} \simeq \tilde{\mathcal{G}}_{-}, \quad \tilde{\mathcal{G}}_{-}^{*} \simeq \tilde{\mathcal{G}}_{+} .
$$

Let now a linear endomorphism $\mathcal{R}: \tilde{\mathcal{G}} \rightarrow \tilde{\mathcal{G}}$ equals $\mathcal{R}=\left(P_{+}-P_{-}\right) / 2$, where, by definitions, $P_{ \pm}: \tilde{\mathcal{G}} \rightarrow \tilde{\mathcal{G}}_{ \pm} \subset \tilde{\mathcal{G}}$ are the projections on the corresponding subspaces $\tilde{\mathcal{G}}_{ \pm} \subset \tilde{\mathcal{G}}$. It is a well known property $[22,20,38,85]$ that for any $\tilde{a}, \tilde{b} \in \tilde{\mathcal{G}}$ the deformed Lie product

$$
[\tilde{a}, \tilde{b}]_{R}:=[\mathcal{R} \tilde{a}, \tilde{b}]+[\tilde{a}, \mathcal{R} \tilde{b}]
$$

satisfies the Jacobi condition and generates on the loop Lie algebra $\tilde{\mathcal{G}}$ a new Lie algebra structure.

Within the classical Adler-Kostant-Symes Lie-algebraic approach, or its $\mathcal{R}$-matrix structure generalization $[22,20,38,85]$, the adjoint loop space $\tilde{\mathcal{G}}^{*}$ is then endowed with the modified Lie-Poisson structure

$$
\{\tilde{l}(\tilde{a}), \tilde{l}(\tilde{b})\}_{\natural}:=\left(\tilde{l} \mid[\tilde{a}, \tilde{b}]_{\mathcal{R}}\right),
$$

for any basic functionals $\tilde{l}(\tilde{a}), \tilde{l}(\tilde{b}) \in D\left(\tilde{\mathcal{G}}^{*}\right)$ subject to which the whole set

$$
I\left(\tilde{\mathcal{G}}^{*}\right)=\left\{\gamma \in D\left(\tilde{\mathcal{G}}^{*}\right) \mid(\tilde{l} \mid[\operatorname{grad} \gamma(\tilde{l}), \tilde{a}])=0_{\natural}, \tilde{a} \in \tilde{\mathcal{G}}^{*}\right\}
$$


of smooth Casimir functionals on $\tilde{\mathcal{G}}^{*}$ is commutative with respect to the deformed Lie-Poisson structure (9.14) on $\tilde{\mathcal{G}}^{*}$, that is $\{\gamma, \mu\}_{\natural}=0_{\natural} \in A_{\natural}$ for all $\gamma, \mu \in I\left(\tilde{\mathcal{G}}^{*}\right)$ and, by definition,

$$
(\tilde{q} \mid \operatorname{grad} \gamma(\tilde{l})):=\left.\frac{d}{d \varepsilon} \gamma(\tilde{l}+\varepsilon \tilde{q})\right|_{\varepsilon=0} .
$$

The latter makes it possible to construct integrable Hamiltonian flows on the associative algebra $A$ as Poissonian flows on the co-adjoint orbits on the adjoint space $\tilde{\mathcal{G}}^{*}$, generated by a suitable loop Lie algebra $\tilde{\mathcal{G}}$ of Casimir gradient elements. Namely, if an element $\tilde{l} \in \tilde{\mathcal{G}}^{*}$ is fixed, the corresponding Hamiltonian flow on $\tilde{\mathcal{G}}^{*}$ subject to the deformed Poisson bracket (9.14) and a Casimir functional $\gamma \in I\left(\tilde{\mathcal{G}}^{*}\right)$ possesses the well known Lax type $[55,65,85]$ representation

$$
d \tilde{l} / d t=\left[P_{+} \operatorname{grad} \gamma(\tilde{l}), \tilde{l}\right],
$$

where $t \in \mathbb{K}$ is a related evolution parameter. The example of this construction and its Lie algebraic properties are discussed in the next Subsection.

\subsection{Kontsevich type integrable systems on unital finitely gene-} rated free associative noncommutative algebras. Let a free unital finitely generated associative non-commutative algebra $A:=\mathbb{K}\left\langle u^{ \pm}, v^{ \pm}\right\rangle$be the corresponding group algebra of a group $G\{u, v\}$, generated by two elements $u, v \in G$. The algebra $A$ is infinite dimensional with the countable basis

$$
\begin{aligned}
L_{A}\left\langle 1, u^{j} v^{s_{1}-j}, v^{j} u^{s_{1}-j}, u^{j} v^{s_{2}-j}\right. & u^{j-k} v^{k-q}, \\
& v^{j} u^{s_{2}-j} v^{j-k} u^{k-q}, \ldots\left|s_{1}, s_{2}, \ldots \in \mathbb{Z}\right\rangle,
\end{aligned}
$$

the related two-dimensional matrix loop Lie algebra $\tilde{\mathcal{G}}=\mathcal{G} \otimes \mathbb{C}\left\{\left\{\lambda, \lambda^{-1}\right\}\right\}$, $\mathcal{G}:=g l(2 ; A)$, is metrized subject to the bi-li near product (9.13) and generated by affine elements

$$
a=\sum_{j=\overline{0,3}} \sigma_{k} \sum_{j \ll \infty} a_{j}^{(k)} \lambda^{j}
$$

with four basis Pauli matrix elements $\sigma_{k} \in g l(2 ; \mathbb{K}), k=\overline{0,3}$, and algebra components $a_{j}^{(k)} \in A, j \ll \infty, k=\overline{0,3}$. The corresponding Casimir functionals $\gamma \in I\left(\tilde{\mathcal{G}}^{*}\right)$ generates a Hamiltonian flow on points $\tilde{l} \in \tilde{\mathcal{G}}^{*}$ with respect to the Poisson bracket (9.14) in the Lax type form (9.15). To analyze this flow in detail, let us put, by definition, that the seed orbit point $\tilde{l} \in \tilde{\mathcal{G}}^{*}$ is given by the following $\lambda$-squared expression

$$
\tilde{l}=\sum_{j=\overline{0,3}} \sum_{k=\overline{0,2}} \sigma^{j} \lambda^{k-3} u_{j}^{(k)},
$$


where

$$
\left\{\sigma^{j} \in g l^{*}(2 ; \mathbb{K}) \mid \operatorname{tr}\left(\sigma^{j} \sigma_{k}\right)=\delta_{k}^{j}, j, k=\overline{0,3}\right\}
$$

is the dual basis of the matrix space $g l^{*}(2 ; \mathbb{K}) \simeq g l(2 ; \mathbb{K})$ and elements

$$
\left\{u_{j}^{(k)} \in A \mid j=\overline{0,3}, k=\overline{0,2}\right\}
$$

are coordinates of some $A$-algebra valued phase space $M_{A}^{(0 \mid 2)}$ in a general position. In particular, we will choose the following dual bases:

$$
\sigma_{0}=\left(\begin{array}{ll}
1 & 0 \\
0 & 1
\end{array}\right), \quad \sigma_{1}=\left(\begin{array}{ll}
0 & 1 \\
0 & 0
\end{array}\right), \quad \sigma_{2}=\left(\begin{array}{ll}
0 & 0 \\
1 & 0
\end{array}\right), \quad \sigma_{3}=\left(\begin{array}{cc}
1 & 0 \\
0 & -1
\end{array}\right)
$$

in $g l(2 ; \mathbb{K})$ and

$$
\sigma^{0}=\left(\begin{array}{cc}
\frac{1}{2} & 0 \\
0 & \frac{1}{2}
\end{array}\right), \quad \sigma^{1}=\left(\begin{array}{cc}
0 & 1 \\
0 & 0
\end{array}\right), \quad \sigma^{2}=\left(\begin{array}{cc}
0 & 0 \\
1 & 0
\end{array}\right), \quad \sigma^{3}=\left(\begin{array}{cc}
\frac{1}{2} & 0 \\
0 & -\frac{1}{2}
\end{array}\right)
$$

in $g l(2 ; \mathbb{K})^{*}$. Moreover, we also will assume that $A$-algebra valued coefficients of the phase space $M_{A}^{(0 \mid 2)}$ and (9.16) are representable subject to the basis of $A$ as

\begin{tabular}{c|ccc}
$\sigma \backslash \lambda$ & $\lambda^{-3}$ & $\lambda^{-2}$ & $\lambda^{-1}$ \\
\hline$\sigma^{0}$ & $u_{0}^{(0)}=1$ & $u_{0}^{(1)}=v+v^{-1}+u+u^{-1}+v^{-1} u^{-1}$ & $u_{0}^{(2)}=0$ \\
$\sigma^{1}$ & $u_{1}^{(0)}=u$ & $u_{1}^{(1)}=v^{-1}$ & $u_{1}^{(2)}=0$ \\
$\sigma^{2}$ & $u_{2}^{(0)}=0$ & $u_{2}^{(1)}=v^{-1} u^{-1}+u^{-1}+1$ & $u_{2}^{(2)}=v$ \\
$\sigma^{3}$ & $u_{3}^{(0)}=-1$ & $u_{3}^{(1)}=-v+v^{-1}+u-u^{-1}-v^{-1} u^{-1}$ & $u_{3}^{(2)}=0$
\end{tabular}

following the result obtained in [96].

As a first important task, we will calculate the corresponding Poisson structure on the related $A$-algebra valued phase space $M_{A}^{(0 \mid 2)}(\tilde{l})$, generated by coefficients, presented in the expression (9.17). To do this, we need to take into account that the phase space $M_{A}^{(0 \mid 2)}(\tilde{l})$, being endowed with the $\mathcal{R}$-modified Poisson structure (9.14), is strongly reduced via the Dirac scheme $[38,78]$ subject to the set

$$
\begin{aligned}
& \Phi:=\left\{\varphi_{1}=u_{0}^{(0)}-1=0, \varphi_{2}=u_{0}^{(2)}=0, \varphi_{3}=u_{2}^{(0)}=0,\right. \\
&\left.\varphi_{4}=u_{3}^{(2)}=0, \varphi_{5}=u_{3}^{(0)}+1=0\right\}
\end{aligned}
$$

of algebraic constraints, imposed on the phase space $M_{A}^{(0 \mid 2)}$. The latter means that the true Poisson structure on the reduced phase space $M_{A}^{(0 \mid 2)}(\tilde{l}):=M_{A}^{(0 \mid 2)} / \Phi$ coincides with the corresponding Dirac type reduction of the $\mathcal{R}$-modified Poisson structure, defined on the full phase space 
$M_{A}^{(0 \mid 2)}$. As a result of simple enough yet cumbersome calculations we arrive at the following Poisson brackets

$$
\{u, v\}_{\natural}=-u v, \quad\{u, u\}_{\natural}=0_{\natural}=\{v, v\}_{\natural}
$$

on the reduced phase space $M_{A}^{(0 \mid 2)}(\tilde{l}) \simeq A:=\mathbb{K}\left\langle u^{ \pm}, v^{ \pm}\right\rangle$.

Having taken as a Hamiltonian operator $h:=r e s \lambda^{2} \operatorname{tr}\left(\tilde{l}^{2}\right) \in I\left(\tilde{\mathcal{G}}^{*}\right)$, one easily obtains the following [51] nonlinear integrable Kontsevich dynamical system

$$
\left.\begin{array}{l}
d u / d t:=\{h, u\}_{\natural}=u v-u v^{-1}-v^{-1} \\
d v / d t:=\{h, v\}_{\natural}=-v u+v u^{-1}+u^{-1}
\end{array}\right\}:=K(u, v)
$$

on the reduced phase space $A=\mathbb{K}\left\langle u^{ \pm}, v^{ \pm}\right\rangle$. Moreover, owing to the Lax type representation (9.14), the Kontsevich dynamical system (9.18) proves to be equivalent to the following matrix commutator equation

$$
d \tilde{l} / d t=[\tilde{l}, p(\tilde{l})]
$$

for any $\lambda \in \mathbb{K}$ in the Lie algebra $\tilde{\mathcal{G}}$, where the $A$-valued matrix

$$
\begin{aligned}
p(\tilde{l})=P_{+} & \operatorname{grad} h(\tilde{l}) / 2=\sigma_{0}\left(v^{-1}-v+u+1\right) / 2+ \\
& +\sigma_{1} \lambda v+\sigma_{2} v^{-1}+\sigma_{3}\left(v^{-1}-v+u-1\right) / 2 \in \tilde{\mathcal{G}} .
\end{aligned}
$$

Taking as Hamiltonian functions the algebraic expressions

$$
h^{(m, n)}:=\operatorname{res} \lambda^{m} \operatorname{tr}\left(\tilde{l}^{n}\right) \in I\left(\tilde{\mathcal{G}}^{*}\right), \quad m, n \in \mathbb{Z},
$$

one can obtain a complete set of $\pi$-commuting to each other conservation laws of the Kontsevich dynamical system (9.18), thus proving its generalized integrability. Moreover, choosing both another group algebra and orbit elements $\tilde{l} \in \tilde{\mathcal{G}}^{*}$, one can construct the same way many other integrable Hamiltonian systems on the associative noncommutative phase space $A$, that is planned to be a topic of a next investigation.

\section{Conclusion}

In this work we succeeded in formal tensor and differential-algebraic reformulating the criteria $[43,90,64]$ for a given differential expression to be Hamiltonian and developed an effective approach to classification of the algebraic Poisson structures lying in the background of the integrable multicomponent Hamiltonian systems. We have devised a simple algorithm allowing to construct new algebraic structures within which the corresponding Hamiltonian operators exist and generate integrable multicomponent dynamical systems. We also showed, as examples, that the well known Balinsky-Novikov algebraic structure, obtained before in $[43,11]$ as a condition for a matrix differential expression to be Hamiltonian, appears within 
the devised approach as a derivation on the adjacent Lie algebra, naturally associated with a suitably constructed differential loop algebra. By means of a direct generalization of this example it is obtained new Lie algebraic relationships, whose background algebraic structures coincide, respectively, with the right Leibniz algebra, introduced in $[23,24,59]$ and with a new Riemann type nonassociative algebra. The constructed Hamiltonian operators describe a wide class of multi-component hierarchies [21, 80] of integrable multicomponent hydrodynamic Riemann type systems. Their reductions appeared to be closely related both to the integrable Camassa-Holm and with the Degasperis-Processi dynamical systems, and are of special interest from the equivalence transformation point of view, devised recently in [95].

Taking into account that the compatible Hamiltonian operators, important for studying integrable multicomponent Hamiltonian systems on functional manifolds, are constructed by means of suitable central extentions of the adjacent weak Lie algebras, determined by the right Leibniz and Riemann type nonassociative and noncommutative algebras, the problem of their description requires a detailed investigation both of their structural properties and finite-dimensional representations of the right Leibniz algebras defined by the corresponding structural constraints. Subject to these important aspects we stopped in the work mostly on the structural properties of the right Leibniz algebras, especially on their derivation algebras and their generalizations. We added also a Supplement in which we revisited the classical Poisson manifolds approach to Hamiltonian operators on functional noncommutative manifolds, as well as presented it simple and natural realization, generated by associative noncommutative group algebra. The latter appeared to be very useful for describing a wide class of new Lax type integrable nonlinear Hamiltonian systems on associative noncommutative algebras, interesting for diverse applications in modern quantum physics.

\section{AcKnowledgements}

The authors are cordially indebted to Anatolij M. Samoilenko, Gerald Goldin, Denis Blackmore, Maciej Błaszak and Błażej Szablikowski for useful comments and remarks, especially for elucidating references, which were very instrumental when preparing a manuscript. They also are indebted to Prof. Anatol Odziewicz for fruitful and instructive discussions during the XXXVIII Workshop on Geometric Methods in Physics held on Juny 30July 06, 2019 in Białowieża, Poland. A.P. thanks also the Departments of Mathematical Sciences of the Rutgers University and the NJIT University of Technology (NJ, USA) for invitations to visit them during the Spring Semester-2019, where there was prepared an essential part of the present 
work. The aknowledgements belong to the Department of Physics, Mathematics and Computer Science of the Cracov University of Technology for a local research grant F-2/370/2018/DS.

The last but not least authors' sincere thanks belong to Referee for her/his very insightful and instrumental comments and suggestions that made the work strongly improved.

\section{REFERENCES}

[1] Ralph Abraham, Jerrold E. Marsden. Foundations of mechanics. Benjamin/Cummings Publishing Co., Inc., Advanced Book Program, Reading, Mass., 1978. Second edition, revised and enlarged, With the assistance of Tudor Raţiu and Richard Cushman.

[2] S. Albeverio, Sh. A. Ayupov, B. A. Omirov. On nilpotent and simple Leibniz algebras. Comm. Algebra, 33(1):159-172, 2005, doi: 10.1081/AGB-200040932.

[3] Massoud Amini, Isamiddin Rakhimov, Seyed Jalal Langari. Enveloping Lie algebras of low dimensional Leibniz algebras. Appl. Math. (Irvine), 2(8):1027-1030, 2011, doi: $10.4236 / \mathrm{am} .2011 .28142$.

[4] V. I. Arnold. Mathematical methods of classical mechanics, volume 60 of Graduate Texts in Mathematics. Springer-Verlag, New York, second edition, 1989, doi: 10.1007/978-1-4757-2063-1. Translated from the Russian by K. Vogtmann and A. Weinstein.

[5] S. Arthamonov. Noncommutative inverse scattering method for the Kontsevich system. Lett. Math. Phys., 105(9):1223-1251, 2015, doi: 10.1007/s11005-015-0779-5.

[6] S. Arthamonov. Modified double Poisson brackets. J. Algebra, 492:212-233, 2017, doi: $10.1016 / j \cdot j a l g e b r a .2017 .08 .025$.

[7] Sh. A. Ayupov, B. A. Omirov. On Leibniz algebras. In Algebra and operator theory (Tashkent, 1997), pages 1-12. Kluwer Acad. Publ., Dordrecht, 1998.

[8] Chengming Bai, Daoji Meng. Addendum: "The classification of Novikov algebras in low dimensions": invariant bilinear forms [J. Phys. A 34 (2001), no. 8, 1581-1594; MR1818753 (2002d:17002)]. J. Phys. A, 34(39):8193-8197, 2001, doi: $10.1088 / 0305-4470 / 34 / 39 / 401$.

[9] Chengming Bai, Daoji Meng. The classification of Novikov algebras in low dimensions. J. Phys. A, 34(8):1581-1594, 2001, doi: 10.1088/0305-4470/34/8/305.

[10] Chengming Bai, Daoji Meng. Transitive Novikov algebras on four-dimensional nilpotent Lie algebras. Internat. J. Theoret. Phys., 40(10):1761-1768, 2001, doi: $10.1023 / \mathrm{A}: 1011968631980$.

[11] A. A. Balinskiı̌, S. P. Novikov. Poisson brackets of hydrodynamic type, Frobenius algebras and Lie algebras. Dokl. Akad. Nauk SSSR, 283(5):1036-1039, 1985.

[12] A. Balinsky, Yu. Burman. Quadratic Poisson brackets and the Drinfeld theory for associative algebras. Lett. Math. Phys., 38(1):63-75, 1996, doi: 10.1007/BF00398299.

[13] A. A. Balinsky, A. I. Balinsky. On the algebraic structures connected with the linear Poisson brackets of hydrodynamics type. J. Phys. A, 26(7):L361-L364, 1993, doi: $10.1088 / 0305-4470 / 26 / 7 / 002$.

[14] A. A. Balinsky, Yu. M. Burman. Quadratic Poisson brackets compatible with an algebra structure. J. Phys. A, 27(18):L693-L696, 1994, doi: 10.1088/0305-4470/27/18/008.

[15] Donald W. Barnes. Faithful representations of Leibniz algebras. Proc. Amer. Math. Soc., 141(9):2991-2995, 2013, doi: 10.1090/S0002-9939-2013-11788-0. 
[16] Chelsie Batten Ray, Alexander Combs, Nicole Gin, Allison Hedges, J. T. Hird, Laurie Zack. Nilpotent Lie and Leibniz algebras. Comm. Algebra, 42(6):2404-2410, 2014, doi: 10.1080/00927872.2012.717655.

[17] A. A. Belavin, V. G. Drinfel' d. Solutions of the classical Yang-Baxter equation for simple Lie algebras. Funktsional. Anal. i Prilozhen., 16(3):1-29, 96, 1982.

[18] A. A. Belavin, V. G. Drinfel' d. The classical Yang-Baxter equation for simple Lie algebras. Funktsional. Anal. i Prilozhen., 17(3):69-70, 1983.

[19] Yves Benoist. Une nilvariété non affine. J. Differential Geom., 41(1):21-52, 1995, doi: $10.4310 / \mathrm{jdg} / 1214456006$.

[20] Denis Blackmore, Anatoliy K. Prykarpatsky, Valeriy Hr. Samoylenko. Nonlinear dynamical systems of mathematical physics. World Scientific Publishing Co. Pte. Ltd., Hackensack, NJ, 2011, doi: 10.1142/9789814327169. Spectral and symplectic integrability analysis.

[21] Denis Blackmore, Yarema A. Prykarpatsky, Nikolai N. Bogolubov, Jr., Anatolij K. Prykarpatski. Integrability of and differential-algebraic structures for spatially 1D hydrodynamical systems of Riemann type. Chaos Solitons Fractals, 59:59-81, 2014, doi: $10.1016 / \mathrm{j}$. chaos.2013.11.012.

[22] Maciej Blaszak. The theory of Hamiltonian and Bi-Hamiltonian systems, page 41-85. Springer Berlin Heidelberg, 1998, doi: 10.1007/978-3-642-58893-8_3.

[23] A. Bloh. On a generalization of the concept of Lie algebra. Dokl. Akad. Nauk SSSR, 165:471-473, 1965.

[24] A. Bloh. Cartan-Eilenberg homology theory for a generalized class of Lie algebras. Soviet Math. Dokl., 8:824-826, 1967.

[25] N. N. Bogolyubov, Jr., A. K. Prykarpatsky. A bilocal periodic problem for SturmLiouville and Dirac operators, and some applications in the theory of nonlinear dynamical systems. I. Ukrain. Mat. Zh., 42(6):794-800, 1990, doi: 10.1007/BF01058917.

[26] Matej Brešar. On the distance of the composition of two derivations to the generalized derivations. Glasgow Math. J., 33(1):89-93, 1991, doi: 10.1017/S0017089500008077.

[27] Dietrich Burde. Affine structures on nilmanifolds. Internat. J. Math., 7(5):599-616, 1996, doi: 10.1142/S0129167X96000323.

[28] Dietrich Burde, Wolfgang Alexander Moens. Periodic derivations and prederivations of Lie algebras. J. Algebra, 357:208-221, 2012, doi: 10.1016/j.jalgebra.2012.02.015.

[29] Roberto Camassa, Darryl D. Holm. An integrable shallow water equation with peaked solitons. Phys. Rev. Lett., 71(11):1661-1664, 1993, doi: 10.1103/PhysRevLett.71.1661.

[30] Ming Chen, Si-Qi Liu, Youjin Zhang. A two-component generalization of the Camassa-Holm equation and its solutions. Lett. Math. Phys., 75(1):1-15, 2006, doi: $10.1007 / \mathrm{s} 11005-005-0041-7$.

[31] A. Degasperis, D. D. Kholm, A. N. I. Khon. A new integrable equation with peakon solutions. Teoret. Mat. Fiz., 133(2):170-183, 2002, doi: 10.1023/A :1021186408422.

[32] A. Degasperis, M. Procesi. Asymptotic integrability. In Symmetry and perturbation theory (Rome, 1998), pages 23-37. World Sci. Publ., River Edge, NJ, 1999.

[33] Ismail Demir, Kailash C. Misra, Ernie Stitzinger. On some structures of Leibniz algebras. In Recent advances in representation theory, quantum groups, algebraic geometry, and related topics, volume 623 of Contemp. Math., pages 41-54. Amer. Math. Soc., Providence, RI, 2014, doi: 10.1090/conm/623/12456.

[34] Irene Dorfman. Dirac structures and integrability of nonlinear evolution equations. Nonlinear Science: Theory and Applications. John Wiley \& Sons, Ltd., Chichester, 1993. 
[35] I. S. Drobotskaya. The Poisson structures related with Lax integrable operator dynamical systems. Preprint/Academy of Sciences of Ukraine, Institute of Mathematics, 93.36:33, 1993.

[36] B. A. Dubrovin, S. P. Novikov. Hamiltonian formalism of one-dimensional systems of the hydrodynamic type and the Bogolyubov-Whitham averaging method. Dokl. Akad. Nauk SSSR, 270(4):781-785, 1983.

[37] B. A. Dubrovin, S. P. Novikov. Poisson brackets of hydrodynamic type. Dokl. Akad. Nauk SSSR, 279(2):294-297, 1984.

[38] L. D. Faddeev, L. A. Takhtajan. Hamiltonian methods in the theory of solitons. Springer Series in Soviet Mathematics. Springer-Verlag, Berlin, 1987, doi: 10.1007/978-3-540-69969-9. Translated from the Russian by A. G. Reyman [A. G. Reı̆man].

[39] Gregorio Falqui. On a Camassa-Holm type equation with two dependent variables. $J$. Phys. A, 39(2):327-342, 2006, doi: 10.1088/0305-4470/39/2/004.

[40] Raúl Felipe, Nancy López-Reyes, Fausto Ongay. R-matrices for Leibniz algebras. Lett. Math. Phys., 63(2):157-164, 2003, doi: 10.1023/A:1023067727095.

[41] A. Fialowski, É. Zs. Mihálka. Representations of Leibniz algebras. Algebr. Represent. Theory, 18(2):477-490, 2015, doi: 10.1007/s10468-014-9505-8.

[42] Alice Fialowski, A. Kh. Khudoyberdiyev, B. A. Omirov. A characterization of nilpotent Leibniz algebras. Algebr. Represent. Theory, 16(5):1489-1505, 2013, doi: $10.1007 / \mathrm{s} 10468-012-9373-z$.

[43] I. M. Gel'fand, I. Ya. Dorfman. Hamiltonian operators and algebraic structures related to them. Functional Analysis and Its Applications, 13(4):248-262, 1980, doi: $10.1007 / \mathrm{bf} 01078363$.

[44] Joł anta Golenia, Maxim V. Pavlov, Ziemowit Popowicz, Anatoliy K. Prykarpatsky. On a nonlocal Ostrovsky-Whitham type dynamical system, its Riemann type inhomogeneous regularizations and their integrability. SIGMA Symmetry Integrability Geom. Methods Appl., 6:Paper 002, 13, 2010, doi: 10.3842/SIGMA.2010.002.

[45] S. Gómez-Vidal, A. Kh. Khudoyberdiyev, B. A. Omirov. Some remarks on semisimple Leibniz algebras. J. Algebra, 410:526-540, 2014, doi: $10.1016 / j \cdot j a l g e b r a .2013 .04 .027$.

[46] D. D. Holm, R. I. Ivanov. Multi-component generalizations of the $\mathrm{CH}$ equation: geometrical aspects, peakons and numerical examples. J. Phys. A, 43(49):492001, 20, 2010, doi: $10.1088 / 1751-8113 / 43 / 49 / 492001$.

[47] John K. Hunter, Ralph Saxton. Dynamics of director fields. SIAM J. Appl. Math., 51(6):1498-1521, 1991, doi: 10.1137/0151075.

[48] N. Jacobson. A note on automorphisms and derivations of Lie algebras. Proc. Amer. Math. Soc., 6:281-283, 1955, doi: 10.2307/2032356.

[49] Nathan Jacobson. Lie algebras. Interscience Tracts in Pure and Applied Mathematics, No. 10. Interscience Publishers (a division of John Wiley \& Sons), New York-London, 1962.

[50] Hyuk Kim. Complete left-invariant affine structures on nilpotent Lie groups. J. Differential Geom., 24(3):373-394, 1986, doi: 10.4310/jdg/1214440553.

[51] Maxim Kontsevich. Formal (non)commutative symplectic geometry. In The Gel'fand Mathematical Seminars, 1990-1992, pages 173-187. Birkhäuser Boston, Boston, MA, 1993.

[52] Maxim Kontsevich. Noncommutative identities. arXiv:1109.2469, 2011. 
[53] Maxim Kontsevich, Alexander L. Rosenberg. Noncommutative smooth spaces. In The Gelfand Mathematical Seminars, 1996-1999, Gelfand Math. Sem., pages 85-108. Birkhäuser Boston, Boston, MA, 2000.

[54] M. Ladra, I. M. Rikhsiboev, R. M. Turdibaev. Automorphisms and derivations of Leibniz algebras. Ukraïn. Mat. Zh., 68(7):933-944, 2016, doi: $10.1007 / \mathrm{s} 11253-016-1277-3$.

[55] Peter D. Lax. Integrals of nonlinear equations of evolution and solitary waves. Comm. Pure Appl. Math., 21:467-490, 1968, doi: 10.1002/cpa.3160210503.

[56] G. Leger. Derivations of Lie algebras. III. Duke Math. J., 30:637-645, 1963, doi: 10.1215/S0012-7094-63-03067-9.

[57] Luen Chau Li, Serge Parmentier. Nonlinear Poisson structures and R-matrices. Comm. Math. Phys., 125(4):545-563, 1989.

[58] André Lichnerowicz. Les variétés de Poisson et leurs algèbres de Lie associées. J. Differential Geometry, 12(2):253-300, 1977, doi: 10.4310/jdg/1214433987.

[59] Jean-Louis Loday. Une version non commutative des algèbres de Lie: les algèbres de Leibniz. Enseign. Math. (2), 39(3-4):269-293, 1993.

[60] Geoffrey Mason, Gaywalee Yamskulna. Leibniz algebras and Lie algebras. SIGMA Symmetry Integrability Geom. Methods Appl., 9:Paper 063, 10, 2013, doi: 10.3842/SIGMA.2013.063.

[61] Alberto Medina Perea. Flat left-invariant connections adapted to the automorphism structure of a Lie group. J. Differential Geometry, 16(3):445-474 (1982), 1981, doi: $10.4310 / \mathrm{jdg} / 1214436223$.

[62] A. V. Mikhailov, V. V. Sokolov. Integrable ODEs on associative algebras. Comm. Math. Phys., 211(1):231-251, 2000, doi: 10.1007/s002200050810.

[63] Wolfgang Alexander Moens. A characterisation of nilpotent Lie algebras by invertible Leibniz-derivations. Comm. Algebra, 41(7):2427-2440, 2013, doi: $10.1080 / 00927872.2012 .659101$.

[64] O. I. Mokhov. Simplekticheskaya $i$ puassonova geometriya na prostranstvakh petel'gladkikh mnogoobraziǔ i integriruemye uravneniya. Sovremennaya Matematika. [Contemporary Mathematics]. Institut Komp'yuternykh Issledovaniŭ, Izhevsk, 2004.

[65] S. Novikov, S. V. Manakov, L. P. Pitaevskiı̌, V. E. Zakharov. Theory of solitons. Contemporary Soviet Mathematics. Consultants Bureau [Plenum], New York, 1984. The inverse scattering method, Translated from the Russian.

[66] A. V. Odesskii, V. N. Rubtsov, V. V. Sokolov. Bi-Hamiltonian ordinary differential equations with matrix variables. Theoret. and Math. Phys., 171(1):442-447, 2012, doi: 10.1007/s11232-012-0043-4. Translation of Teoret. Mat. Fiz. 171 (2012), no. $1,26-32$.

[67] Alexander Odesskii, Vladimir Rubtsov, Vladimir Sokolov. Double Poisson brackets on free associative algebras. In Noncommutative birational geometry, representations and combinatorics, volume 592 of Contemp. Math., pages 225-239. Amer. Math. Soc., Providence, RI, 2013, doi: 10.1090/conm/592/11861.

[68] Walter Oevel. Dirac constraints in field theory: lifts of Hamiltonian systems to the cotangent bundle. J. Math. Phys., 29(1):210-219, 1988, doi: 10.1063/1.528175.

[69] Walter Oevel. $R$ structures, Yang-Baxter equations, and related involution theorems. J. Math. Phys., 30(5):1140-1149, 1989, doi: 10.1063/1.528333.

[70] Peter J. Olver. Applications of Lie groups to differential equations, volume 107 of Graduate Texts in Mathematics. Springer-Verlag, New York, second edition, 1993, doi: 10.1007/978-1-4612-4350-2. 
[71] Peter J. Olver, Vladimir V. Sokolov. Integrable evolution equations on associative algebras. Comm. Math. Phys., 193(2):245-268, 1998, doi: 10.1007/s002200050328.

[72] B. A. Omirov. On derivations of filiform Leibniz algebras. Mat. Zametki, 77(5):733-742, 2005, doi: $10.1007 /$ s11006-005-0068-1.

[73] J. Marshall Osborn. Novikov algebras. Nova J. Algebra Geom., 1(1):1-13, 1992.

[74] Maxim V. Pavlov. The Gurevich-Zybin system. J. Phys. A, 38(17):3823-3840, 2005, doi: $10.1088 / 0305-4470 / 38 / 17 / 008$.

[75] Ziemowit Popowicz, Anatoliy K. Prykarpatsky. The non-polynomial conservation laws and integrability analysis of generalized Riemann type hydrodynamical equations. Nonlinearity, 23(10):2517-2537, 2010, doi: 10.1088/0951-7715/23/10/010.

[76] A. K. Prikarpatskiı̌, N. N. Bogolyubov. A bilocal periodic problem for Sturm-Liouville and Dirac differential operators, and some applications in the theory of nonlinear dynamical systems. Dokl. Akad. Nauk SSSR, 310(1):29-32, 1990.

[77] Anatoliy K. Prykarpatsky, Orest D. Artemovych, Ziemowit Popowicz, Maxim V. Pavlov. Differential-algebraic integrability analysis of the generalized Riemann type and Korteweg-de Vries hydrodynamical equations. J. Phys. A, 43(29):295205, 13, 2010, doi: $10.1088 / 1751-8113 / 43 / 29 / 295205$.

[78] Anatoliy K. Prykarpatsky, Ihor V. Mykytiuk. Algebraic integrability of nonlinear dynamical systems on manifolds, volume 443 of Mathematics and its Applications. Kluwer Academic Publishers Group, Dordrecht, 1998, doi: 10.1007/978-94-011-4994-5. Classical and quantum aspects.

[79] Anatoliy K. Prykarpatsky, Mykola M. Prytula. The gradient-holonomic integrability analysis of a Whitham-type nonlinear dynamical model for a relaxing medium with spatial memory. Nonlinearity, 19(9):2115-2122, 2006, doi: $10.1088 / 0951-7715 / 19 / 9 / 007$.

[80] Yarema A. Prykarpatsky, Orest D. Artemovych, Maxim V. Pavlov, Anatoliy K. Prykarpatsky. Differential-algebraic and bi-Hamiltonian integrability analysis of the Riemann hierarchy revisited. J. Math. Phys., 53(10):103521, 20, 2012, doi: $10.1063 / 1.4761821$.

[81] Yarema A. Prykarpatsky, Orest D. Artemovych, Maxim V. Pavlov, Anatoliy K. Prykarpatsky. Differential-algebraic and bi-Hamiltonian integrability analysis of the Riemann hierarchy revisited. J. Math. Phys., 53(10):103521, 20, 2012, doi: $10.1063 / 1.4761821$.

[82] I. S. Rakhimov, Al-Nashri Al-Hossain. On derivations of low-dimensional complex Leibniz algebras. JP J. Algebra Number Theory Appl., 21(1):69-81, 2011.

[83] I. S. Rakhimov, K. K. Masutova, B. A. Omirov. On derivations of semisimple Leibniz algebras. Bull. Malays. Math. Sci. Soc., 40(1):295-306, 2017, doi: $10.1007 / \mathrm{s} 40840-015-0113-5$.

[84] Isamiddin S. Rakhimov, Al-Hossain Al-Nashri. On derivations of some classes of Leibniz algebras. J. Gen. Lie Theory Appl., 6:Art. ID G120501, 12, 2012, doi: $10.4303 / \mathrm{jglta} /$ G120501.

[85] M. A. Reyman, M Semenov-Tian-Shansky. Integrable systems. 2003 (in Russian).

[86] Gian-Carlo Rota. Baxter algebras and combinatorial identities. I. Bull. Amer. Math. Soc., 75:325-329, 1969, doi: 10.1090/S0002-9904-1969-12156-7.

[87] Gian-Carlo Rota. Baxter algebras and combinatorial identities. II. Bull. Amer. Math. Soc. 75 (1969), 325-329; ibid., 75:330-334, 1969, doi: 10.1090/S0002-9904-1969-12158-0.

[88] R. D. Schafer. Inner derivations of non-associative algebras. Bull. Amer. Math. Soc., 55:769-776, 1949, doi: 10.1090/S0002-9904-1949-09281-9. 
[89] M. A. Semenov-Tyan-Shanskiı̌. What a classical $R$-matrix is. Funktsional. Anal. $i$ Prilozhen., 17(4):17-33, 1983.

[90] A. Sergyeyev. A simple way of making a Hamiltonian system into a bi-Hamiltonian one. Acta Appl. Math., 83(1-2):183-197, 2004, doi: $10.1023 / B$ :ACAP. 0000035597.06308 .8 a.

[91] Ian A. B. Strachan, Błażej M. Szablikowski. Novikov algebras and a classification of multicomponent Camassa-Holm equations. Stud. Appl. Math., 133(1):84-117, 2014, doi: $10.1111 /$ sapm. 12040.

[92] Shigeaki Tôgô. On the derivation algebras of Lie algebras. Canadian J. Math., 13:201216, 1961, doi: 10.4153/CJM-1961-017-8.

[93] Shigeaki Tôgô. Outer derivations of Lie algebras. Trans. Amer. Math. Soc., 128:264276, 1967, doi: 10.2307/1994323.

[94] Michel Van den Bergh. Double Poisson algebras. Trans. Amer. Math. Soc., 360(11):5711-5769, 2008, doi: 10.1090/S0002-9947-08-04518-2.

[95] Olena O. Vaneeva, Roman O. Popovych, Christodoulos Sophocleous. Equivalence transformations in the study of integrability. Physica Scripta, 89(3):38003, Feb 2014, doi: $10.1088 / 0031-8949 / 89 / 03 / 038003$.

[96] Thomas Wolf, Olga Efimovskaya. On integrability of the Kontsevich non-abelian ODE system. Lett. Math. Phys., 100(2):161-170, 2012, doi: 10.1007/s11005-011-0527-4.

Received: August 13, 2019, accepted: December 1, 2019.

Orest D. Artemovych

The Department of Algebra at the Krakow University of Technology, Krakow, POLAND

Email: artemo@usk.pk.edu.pl

Alexander A. Balinsky

The Mathematics Institute at the Cardiff University, Cardiff CF24 4AG, Great BRITAIN

Email: BalinskyA@cardiff.ac.uk

Anatolij K. Prykarpatski

Department of Physics, Mathematics and Computer Science at the Cracov University of Technology, Krakow, Poland

Email: pryk.anat@cybergal.com 